\title{
Covariance and time regained in canonical general relativity
}

\author{
I. Kouletsis \\ February 2008
}

\begin{abstract}
Canonical vacuum gravity is expressed in generally-covariant form in order that spacetime diffeomorphisms be represented within its equal-time phase space. In accordance with the principle of general covariance and ideas developed within history phase space formalisms in Refs. [1]-[4], the time mapping $\mathbf{T}: \mathcal{M} \rightarrow \mathbb{R}$ and the space mapping $\mathbf{X}: \mathcal{M} \rightarrow \Sigma$ that define the Dirac-ADM foliation are incorporated into the framework of the Hilbert variational principle. The resulting canonical action encompasses all individual Dirac-ADM actions, corresponding to different choices of foliating vacuum spacetimes by spacelike hypersurfaces. The equal-time phase space $\mathcal{P}=\left\{g_{i j}, p^{i j}, Y^{\alpha}, P_{\alpha}\right\}$ includes the embeddings $Y^{\alpha}$ and their conjugate momenta $P_{\alpha}$. It is constrained by eight first-class constraints. The constraint surface $\mathcal{C}$ is determined by the super-Hamiltonian and super-momentum constraints of vacuum gravity and the vanishing of the embedding momenta. Deformations of the time and space mappings, $\delta \mathbf{T}$ and $\delta \mathbf{X}$, and spacetime diffeomorphisms, $V \in$ LDiff $\mathcal{M}$, induce symplectic diffeomorphisms of $\mathcal{P}$. While the generator $\mathcal{D}_{(\delta \mathbf{T}, \delta \mathbf{X})}$ of deformations depends on all eight constraints, the generator $\mathcal{D}_{V}$ of spacetime diffeomorphisms depends only on the embedding momentum constraints. As a result, spacetime observables, namely, dynamical variables $F$ on $\mathcal{P}$ that are invariant under spacetime diffeomorphisms, $\left.\left\{F, \mathcal{D}_{V}\right\}\right|_{\mathcal{C}}=0$, are not necessarily invariant under the deformations of the mappings, $\left.\left\{F, \mathcal{D}_{(\delta \mathbf{T}, \delta \mathbf{X})}\right\}\right|_{\mathcal{C}} \neq 0$, nor are they constants of the motion, $\left.\left\{F, \int d^{3} x \mathcal{H}\right\}\right|_{\mathcal{C}} \neq 0$. Dirac observables form only a subset of spacetime observables that are invariant under the transformations of $\mathbf{T}$ and $\mathbf{X}$ and do not evolve in time. In this generally-covariant framework, the conventional interpretation of the canonical theory, due to Bergmann and Dirac, amounts to postulating that the transformations of the reference system $(\mathbf{T}, \mathbf{X})$ have no measurable consequences; i.e., that all first-class constraints generate gauge transformations. If this postulate is not deemed necessary, canonical gravity admits no classical problem of time.
\end{abstract}




\section{Introduction}

\subsection{General covariance, determinism and the problem of evolution}

The variational principle for general relativity, with or without sources, introduces a fourdimensional manifold $\mathcal{M}$ and an action functional $S[\Psi]$ on $\mathcal{M}$. The principle of general covariance demands that all fields $\boldsymbol{\Psi}$ be subject to variation in the action functional and satisfy generally-covariant field equations. In the case of the vacuum theory, where only the metric field is present on the spacetime manifold, the set of solutions consists of all distinct metric fields $\mathbf{G}$ on $\mathcal{M}$ that satisfy the vacuum Einstein equations. These solutions do not all correspond to physically distinct states of the system. Considering that the manifold points are physically indistinguishable prior to introducing the fields on $\mathcal{M}$, any two solutions that can be brought into coincidence by an element of the group Diff $\mathcal{M}$ are regarded as representations of the same physical state. The group Diff $\mathcal{M}$ is treated as the gauge group of the theory, and each physical state is identified with an equivalence class $\{\mathbf{G}\}$ of Diff $\mathcal{M}$-related solutions on $\mathcal{M}$. The set $\Gamma$ of all such equivalence classes constitutes the set of physically distinct states of the system.

In the canonical formalism, initiated by Dirac [5] and Arnowitt, Deser and Misner [6], the same physical conclusion can be drawn by considering the initial-value problem. General relativity is not a deterministic dynamical system in the strict sense. A characteristic of its canonical formulation is that a given set of instantaneous data at an initial time $t_{1}$ may evolve, via different choices of lapse and shift, to many different sets of such data at a later time $t_{2}$. Nevertheless, a well-posed initial-value problem arises if it is stipulated that all these sets of evolved data characterise the same physical situation [7]-8]. Within the framework of the Dirac-ADM phase space $\mathcal{P}=\left\{\left(g_{i j}, p^{i j}\right)\right\}$, each set of permissible data $\left(g_{i j}(x), p^{i j}(x)\right)$ on a given hypersurface defines a point on the constraint surface $\mathcal{C} \subset \mathcal{P}$, where $\mathcal{C}$ is determined by the first-class constraints. All points in $\mathcal{C}$ to which an initial point can evolve via arbitrary choices of lapse and shift lie in an orbit of the Hamiltonian vector field generated by the first-class constraints. The set $\Delta$ of such distinct orbits in $\mathcal{C}$, equipped with an induced symplectic form, constitutes the so-called reduced phase space of the theory. This set can be brought into a one-to-one correspondence with the set $\Gamma$ of Diff $\mathcal{M}$-classes of solutions on $\mathcal{M}$ [9].

In this way, the original classification of physical states according to the set $\Gamma$ is recovered, and the inability to physically distinguish between evolved data in the canonical theory may be attributed to the invariance of the spacetime action under Diff $\mathcal{M}$. In addition, the bijective correspondence between the sets $\Gamma$ and $\Delta$ allows the physical observables of the theory to be perceived either as functions on $\Delta$, the so-called Dirac observables, or as functions on $\Gamma$, which may be referred to as spacetime observables. In either case, the physical observables remain invariant under the dynamical evolution generated by the Hamiltonian, a fact which implies that this evolution is not measurable. Only symmetries of the reduced phase space, i.e., symplectic transformations of $\Delta$, and equivalently of $\Gamma$, can be contemplated as being measurable [10]. Even if such symmetries are discovered in general relativity, global obstructions are expected 
to arise in the phase space [11] which may prohibit such symmetries from being interpreted as generators of the evolution of the system in physical time. This problem of evolution may be regarded as the classical core of the problem of time of quantum gravity.

\subsection{The missing representations of the group Diff $\mathcal{M}$}

Of particular relevance to the problem of evolution is the way in which the group Diff $\mathcal{M}$ is considered to act on the phase space of general relativity, and the connection between this action and the dynamical evolution generated by the Hamiltonian. A peculiar feature of the Dirac-ADM formalism is that, despite the bijective correspondence between the sets $\Gamma$ and $\Delta$, the Diff $\mathcal{M}$-invariance of the spacetime action is reflected only indirectly in the first-class constraints. More precisely, although the canonical transformations generated by the Hamiltonian can be linked to the diffeomorphisms of the spacetime manifold $\mathcal{M}$, the Lie algebra of Diff $\mathcal{M}$ cannot be mapped onto the Poisson bracket algebra of the super-Hamiltonian and super-momentum constraints. This inability to recover the action of Diff $\mathcal{M}$ directly within the conventional canonical framework is not only noteworthy from the conceptual point of view, but also contributes to the problems that hinder the canonical quantisation of gravity.

The cause of this difficulty was diagnosed by Isham and Kuchař [12]. The absence from the conventional phase space $\mathcal{P}=\left\{\left(g_{i j}, p^{i j}\right)\right\}$ of the embedding mappings $Y: \Sigma \rightarrow \mathcal{M}$ that connect the spacetime manifold $\mathcal{M}$ with the space manifold $\Sigma$ renders the direct canonical description of spacetime objects impossible, and leads to the loss of the representations of Diff $\mathcal{M}$. In order to recover the action of Diff $\mathcal{M}$ within the canonical framework, this missing link must be re-established, and the gravitational configuration space must be extended by the space of embeddings from $\Sigma$ to $\mathcal{M}$. This was achieved in Ref. [12] by parameterising the Dirac-ADM action.

The process of parameterisation is tantamount to viewing the lapse function and the shift vector as functionals of the embedding mapping $Y: \Sigma \rightarrow \mathcal{M}$, and then varying $Y$ in the action. When applied to a generally-covariant system such as general relativity, this procedure requires that four of the components of the spacetime metric be limited by coordinate conditions with respect to the foliation structure. The coordinate conditions are needed in order that the lapse function and the shift vector can indeed be regarded as functionals of the embedding variable $Y$, and not as variables on their own. In addition, these conditions ensure a well-posed initialvalue problem. Without them, the spacetime metric built by the canonical dynamical evolution would be determined only up to a spacetime diffeomorphism [13].

As a result of limiting in Ref. [12 the spacetime metric by the coordinate conditions, the original super-Hamiltonian and super-momentum constraints get suspended, and new, modified, constraints arise. In the resulting phase space $\left\{\left(g_{i j}, p^{i j}, Y^{\alpha}, P_{\alpha}\right)\right\}$, augmented by the embeddings $Y^{\alpha}(x)$ and their conjugate momenta $P_{\alpha}(x)$, a direct correspondence between the spacetime and the canonical descriptions emerges. This is attested via the construction of a homomorphic mapping from the Lie algebra of Diff $\mathcal{M}$ into the Poisson bracket algebra of the dynamical 
variables on the extended phase space.

Viewed from the perspective of a variational principle, the procedure of breaking the invariance of general relativity by coordinate conditions and restoring it by parameterisation can be associated with the coupling of gravity to matter fields. Kuchař and Torre [14] derived the modified constraints of Isham and Kuchař from an appropriate action functional, and recognised the new terms as the energy-momentum density of a non-rotating, heat-conducting, incoherent dust. Other coordinate conditions lead to different constraint structures, some of which have been investigated in Refs. [15]-[21].

\subsection{Aim, motivation and main concept}

In this paper, a reformulation of the canonical method is considered, that permits the representation of the Lie algebra of $\operatorname{Diff} \mathcal{M}$ within a suitable equal-time phase space for vacuum general relativity, without abandoning the standard constraints of this theory. The proposed formalism relies upon ideas and techniques that were developed in collaboration with K. Kuchař in Ref. [1] and yields results that are, in certain ways, parallel to the results of Savvidou [2]-[4], derived within the context of the History Projection Operator 11 formalism for general relativity.

From a technical point of view, the only difference between the present formulation and the conventional formulation of Dirac and ADM is that the foliation is modelled as a variable, and is incorporated into the framework of the Hilbert variational principle. Such an approach is actuated by the desire to harmonise the canonical action with the principle of general covariance, and the recognition of the fact that, strictly speaking, this action is an extension of the Hilbert action. This is because, by construction, the canonical action requires a time foliation of $\mathcal{M}$ by spacelike hypersurfaces to be introduced into general relativity as an additional geometric element.

Thereby, the notion of time is distinguished from that of a spacetime coordinate and becomes dependent upon the spacetime metric G. Time is represented by a global scalar mapping $\mathbf{T}: \mathcal{M} \rightarrow \mathbb{R}$ from the spacetime manifold $\mathcal{M}$ to a one-dimensional time manifold $\mathbb{R}$ which has the topology of the open line. The gradient $\mathbf{T}_{, \alpha}$ of this mapping is required to be timelike with respect to $\mathbf{G}$. Accordingly, each choice of $\mathbf{T}$ represents a foliation of $\mathcal{M}$ by spacelike hypersurfaces. On each such hypersurface, the notion of space is represented by another metricdependent mapping $\mathbf{X}: \mathcal{M} \rightarrow \Sigma$, whose gradients $\mathbf{X}_{, \alpha}^{i}$ are required to be spacelike.

In order that the canonical theory be cast in generally-covariant form, all fields upon which it is based must conform to the principle of general covariance. That is, they must be subject to variation in the action functional and satisfy generally-covariant field equations. In particular, field equations must be satisfied by $\mathbf{T}$ and $\mathbf{X}$. These equations must enforce the timelike and spacelike character of the gradients of these variables, but must otherwise leave $\mathbf{T}$ and

\footnotetext{
${ }^{1}$ The Consistent Histories interpretation of quantum theory was initiated by Griffiths [22] and developed by Omnès [23]-[26], Gell-Mann and Hartle [27]-31] and Isham [32]; see also [33]-38].
} 
$\mathbf{X}$ undetermined in order to respect the arbitrariness of the spacelike foliation. As a result, a generally-covariant canonical action must necessary involve a greater number of non-dynamical variables than the Hilbert action. This causes the breaking of the bijective correspondence between its sets $\Gamma$ and $\Delta$ and, therefore, has repercussions for the functions defined on these sets; namely, the spacetime observables and the Dirac observables.

As it is evident, the breaking of this correspondence is a crucial property of the covariant canonical action. In general, the sets $\Gamma$ and $\Delta$ reveal different aspects of a generally-covariant theory: on the one hand, the set $\Gamma$ is derived from the set of solutions by eliminating only the freedom associated with Diff $\mathcal{M}$. On the other hand, the set $\Delta$ is derived from the set of solutions by eliminating the freedom associated with the first-class constraints. In an arbitrary generally-covariant framework, this latter freedom may be wider than the former, because it depends upon the number of non-dynamical variables present in the action; i.e., variables that are left undetermined by the variational principle. In our particular case of interest, after the non-dynamical variables $\mathbf{T}$ and $\mathbf{X}$ are incorporated into the framework of the Hilbert variational principle, the dynamical content of the resulting action, expressed by the set $\Delta$, remains unaffected. However, the set $\Gamma$ of Diff $\mathcal{M}$-classes of solutions is extended by the presence of these arbitrary fields. The set $\Delta$ becomes a subset of $\Gamma$, and the Dirac observables form only a subset of the spacetime observables. Since it is just this subset that weakly commutes with the Hamiltonian, the evolution of the spacetime observables is, in general, non-trivial.

In addition, the breaking of the bijective correspondence between the sets $\Gamma$ and $\Delta$ is reflected within the equal-time phase space $\mathcal{P}$ in the doubling of the first-class constraints. Thus, it becomes possible in the covariant canonical formalism to identify which constraints arise due to the diffeomorphism invariance of the spacetime action and which arise due to the nondynamical character of the foliation. This lays the foundations for, firstly, representing the Lie algebra of spacetime diffeomorphisms by symplectic diffeomorphisms of $\mathcal{P}$ and, secondly, for separating the canonical transformations generated by spacetime diffeomorphisms from those generated by the deformations of the foliation. In comparison, the bijection between the sets $\Gamma$ and $\Delta$ in the Dirac-ADM formalism leads to the entanglement of distinct concepts and the loss of general covariance, while the preservation of this bijection via the coordinate conditions causes the suspension of the vacuum constraints in the covariant framework of Isham and Kuchař.

\subsection{The formalism}

A general procedure for incorporating the foliation into the variational principle of a generallycovariant theory was developed in Ref. [1. It was designed originally for the purpose of representing spacetime diffeomorphisms in the history phase space of an arbitrary generallycovariant system, modelled in Ref. [1] by the Bosonic string. This procedure respects the distinction between the sets $\Gamma$ and $\Delta$, so it only needs to be adapted to the circumstances of the gravitational theory. Thus, in the same spirit, a time variable $\mathbf{T}: \mathcal{M} \rightarrow \mathbb{R}$ and a space 
variable $\mathbf{X}: \mathcal{M} \rightarrow \Sigma$ are incorporated into the Hilbert action as additional variable fields. While the mapping $\mathbf{T}$ describes a slicing of the spacetime manifold by spacelike hypersurfaces, namely, a time foliation, the mapping $\mathbf{X}$ describes a congruence of timelike reference worldlines, namely, a reference frame. The product mapping $\mathbf{T} \times \mathbf{X}: \mathcal{M} \rightarrow \mathbb{R} \times \Sigma$ is inverse to the foliation mapping $Y: \mathbb{R} \times \Sigma \rightarrow \mathcal{M}$.

The variables $\mathbf{T}$ and $\mathbf{X}$ are coupled to the spacetime metric $\mathbf{G}$. This coupling preserves the vacuum Einstein equations and also ensures that, at the level of the solutions, the time foliation is spacelike, and the reference frame is timelike, with respect to $\mathbf{G}$. Apart from these essential restrictions, the variables $\mathbf{T}$ and $\mathbf{X}$ are left undetermined by the variational principle in order to comply with the arbitrariness of the foliation. The resulting set of solutions $\left\{\mathbf{G}_{\alpha \beta}, \mathbf{T}, \mathbf{X}^{i}\right\}$ incorporates the content of all individual Dirac-ADM actions in the sense that it includes all causal reference systems that can be associated with each vacuum spacetime. All the fields in this extended action functional transform covariantly under the diffeomorphisms of $\mathcal{M}$, so the general covariance of the formalism remains manifest.

As in Ref. [1], the transition from the spacetime action to its Lagrangian counterpart on $\mathbb{R} \times$ $\Sigma$ is conceived as a one-to-one transformation from the set of spacetime variables $\left\{\mathbf{G}_{\alpha \beta}, \mathbf{T}, \mathbf{X}^{i}\right\}$ to the set $\left\{g_{i j}, N, N^{i}, Y^{\alpha}\right\}$ of induced variables on $\mathbb{R} \times \Sigma$. This transformation is followed by a Legendre transformation, which involves the foliation field $Y$. Since the spacetime and the canonical frameworks remain interlinked, all symmetries of the original field equations on $\mathcal{M}$ are transferred to the canonical theory. This provides the basis for studying the transformations induced on the canonical fields $\left\{g_{i j}, p^{i j}, N, N^{i}\right\}$ by the diffeomorphisms of $\mathcal{M}$, as well as by the deformations of the mappings $\mathbf{T}$ and $\mathbf{X}$.

Resembling the covariant formulation of Isham and Kuchař, the equal-time phase space $\mathcal{P}=\left\{\left(g_{i j}, p^{i j}, Y^{\alpha}, P_{\alpha}\right)\right\}$ includes the embeddings $Y^{\alpha}(x)$ and their conjugate momenta $P_{\alpha}(x)$. However, it is now constrained by eight first-class constraints. The constraint surface $\mathcal{C}$ is determined by the standard super-Hamiltonian and super-momentum constraints of vacuum gravity, $H=0$ and $H_{i}=0$, and the vanishing of the embedding momenta, $P_{\alpha}=0$. The Hamiltonian $\int d^{3} x \mathcal{H}$ is a linear functional of these eight first-class constraints, $\mathcal{H}:=N H+$ $N^{i} H_{i}+\Lambda^{\alpha} P_{\alpha}$.

\subsection{Summary of results}

The Hamiltonian $\int d^{3} x \mathcal{H}$ is regarded as the generator of solutions rather than of symmetries; that is, its primary role is considered to be the creation of solutions from permissible instantaneous data. Symmetries of the field equations then act on these solutions. Symmetries are generated by infinitesimal transformations of the field variables that preserve the linearisation of the field equations provided that these equations hold. Each symmetry defines a mapping of solutions to solutions. Key symmetries are induced by the diffeomorphisms of the manifolds $\mathcal{M}$ and the transformations of the mappings $\mathbf{T}$ and $\mathbf{X}$.

The diffeomorphisms of $\mathcal{M}$ do not act on solutions in the same way as the transformations 
of $\mathbf{T}$ and $\mathbf{X}$ do. Under the action of Diff $\mathcal{M}$, the spacetime metric $\mathbf{G}$ and the mappings $\mathbf{T}$ and $\mathbf{X}$ transform covariantly. This implies, in particular, that the spacelike character of the time foliation and the timelike character of the reference frame are respected. The foliation variable $Y$, being inverse to $\mathbf{T} \times \mathbf{X}$, is transformed arbitrarily by Diff $\mathcal{M}$, but the fields $g, p, N$ and $N^{i}$ remain unchanged. In contrast, under the transformations of the mappings $\mathbf{T}$ and $\mathbf{X}$, the spacetime metric $\mathbf{G}$ is left, by definition, unchanged, but the fields $g, p, N, N^{i}$ and $Y$ are all transformed.

Special kinds of transformations of $\mathbf{T}$ and $\mathbf{X}$ are induced by the diffeomorphisms of the manifolds $\mathbb{R}$ and $\Sigma$. These move individual hypersurfaces and individual worldlines, but they keep the time foliation and the reference frame fixed; i.e., the final collection of hypersurfaces and worldlines is the same as the original. On account of this, the spacelike character of the time foliation and the timelike character of the reference frame, with respect to the unchanged $\mathbf{G}$, are preserved. This is not the case for more general transformations of $\mathbf{T}$ and $\mathbf{X}$, unless these transformations are allowed to depend fully upon solutions. Then it is indeed possible to consider generalised symmetries $\delta \mathbf{T}[\mathbf{G}, \mathbf{T}, \mathbf{X}]$ and $\delta \mathbf{X}[\mathbf{G}, \mathbf{T}, \mathbf{X}]$ that sustain the compatibility between the mappings $\mathbf{T}, \mathbf{X}$ and the unchanged $\mathbf{G}$.

Within the framework of the extended phase space $\mathcal{P}$, solutions are visualised as curves lying in the subspace $\mathbb{R} \times \mathcal{C}$ of $\mathbb{R} \times \mathcal{P}$. Symmetries of the field equations are acting on these curves. The special transformations induced on $\mathbb{R} \times \mathcal{P}$ by infinitesimal time diffeomorphisms $w \in \mathrm{LDiffR}$ and infinitesimal space diffeomorphisms $u \in \mathrm{LDiff} \Sigma$ are generated, respectively, by the dynamical variables $\mathcal{D}_{w}=-\int d^{3} x w \mathcal{H}$ and $\mathcal{D}_{u}=-\int d^{3} x u^{i}\left(H_{i}+P_{\alpha} Y_{, i}^{\alpha}\right)$. The more general transformations induced on $\mathbb{R} \times \mathcal{P}$ by the symmetries $\delta \mathbf{T}[\mathbf{G}, \mathbf{T}, \mathbf{X}]$ and $\delta \mathbf{X}[\mathbf{G}, \mathbf{T}, \mathbf{X}]$ are generated by the functional $\mathcal{D}_{(\delta \mathbf{T}, \delta \mathbf{X})}=-\int d^{3} x\left(\delta \mathbf{T} \mathcal{H}-\delta \mathbf{X}^{i}\left(H_{i}+P_{\alpha} Y_{, i}^{\alpha}\right)\right)$. This reduces to the generator $\mathcal{D}_{w}$ in the case where $\delta \mathbf{T}=w(\mathbf{T})$ and $\delta \mathbf{X}=0$, and to the generator $\mathcal{D}_{u}$ in the case where $\delta \mathbf{T}=0$ and $\delta \mathbf{X}=u(\mathbf{X})$. Analogous functionals can be constructed within the Dirac-ADM phase space $\left\{g_{i j}, p^{i j}\right\}$.

On the other hand, the symmetries induced on $\mathbb{R} \times \mathcal{P}$ by infinitesimal spacetime diffeomorphisms $V \in$ LDiff $\mathcal{M}$ are generated by a dynamical variable that has no counterpart in the conventional phase space. This is the variable $\mathcal{D}_{V}=\int d^{3} x P_{\alpha} V^{\alpha}(Y)$, which depends solely on the embedding variables and the vector field $V$. This functional provides an anti-homomorphic mapping of vector fields in the Lie algebra LDiff $\mathcal{M}$ into the Poisson bracket algebra on the phase space $\mathcal{P}$; i.e., a representation of spacetime diffeomorphisms by symplectic diffeomorphisms of the phase space.

The structure of the generators $\mathcal{D}_{(\delta \mathbf{T}, \delta \mathbf{X})}$ and $\mathcal{D}_{V}$ reveals two facts about canonical general relativity that lay unexpressed within the conventional canonical formalism. First, the general covariance of the theory is not reflected in the super-Hamiltonian and super-momentum constraints but, instead, in the embedding momentum constraints. Second, the orbits of the generators $\mathcal{D}_{V}$ and $\int d^{3} x \mathcal{H}$ on the phase space $\mathcal{P}$ are distinct, in accordance with the set $\Delta$ being a subset of $\Gamma$. This eliminates any possibility of identifying the Hamiltonian functional 
$\int d^{3} x \mathcal{H}$ with the generator of spacetime diffeomorphisms, in agreement with Kuchař's analysis of this issue in Ref. [39].

Although this distinct role of the Hamiltonian, as opposed to the role of spacetime diffeomorphisms, cannot find an unambiguous mathematical expression within the standard phase space $\left\{\left(g_{i j}, p^{i j}\right)\right\}$ of vacuum gravity, it has been enacted in formulations based on history phase spaces. In Ref. [2], history representations of both the Lie algebra of Diff $\mathcal{M}$ and the Dirac algebra of the constraints are constructed within the context of the History Projection Operator formalism for general relativity. The foliation is introduced as a parameter in the formalism and satisfies an equivariance condition [3]-[4]. The invariance of the canonical action under Diff $\mathcal{M}$ was thereby established, and the connection between this fact and the problem of time was studied. The issue of the history quantisation of a spacelike foliation was also analysed - see Ref. [40].

Alternative history representations of Diff $\mathcal{M}$ were constructed in Ref. [1] in the context of the history phase space of the Bosonic string. The equal-time formalism considered here has inherited several features from that history formalism; among them, the incorporation of the mappings $\mathbf{T}$ and $\mathbf{X}$ in the variational principle, which makes the correspondence between the sets $\Gamma$ and $\Delta$ many-to-one. This leads to the enrichment of the notion of instantaneous observables and calls for the revision of their dynamical evolution. As anticipated, two kinds of observables arise on the equal-time phase space $\mathcal{P}$ of the covariant canonical action: spacetime observables and Dirac observables.

The spacetime observables are dynamical variables $F$ on $\mathcal{P}$ that commute on the constraint surface $\mathcal{C}$ with the generator of spacetime diffeomorphisms, $\left.\left\{F, \mathcal{D}_{V}\right\}\right|_{\mathcal{C}}=0$. While such functionals weakly commute with the embedding momentum constraints, they do not necessarily weakly commute with the super-Hamiltonian and super-momentum constraints. As a result, they are not necessarily invariant under the deformations of the mappings, $\left.\left\{F, \mathcal{D}_{(\delta \mathbf{T}, \delta \mathbf{X})}\right\}\right|_{\mathcal{C}} \neq 0$, nor are they constants of the motion, $\left.\left\{F, \int d^{3} x \mathcal{H}\right\}\right|_{\mathcal{C}} \neq 0$. On the other hand, the Dirac observables weakly commute with all eight first-class constraints, and hence also with $\int d^{3} x \mathcal{H}$. These are invariant under both the diffeomorphisms of $\mathcal{M}$ and the transformations of the mappings $\mathbf{T}$ and $\mathbf{X}$, and form a subset of spacetime observables that remain frozen in time. While the spacetime observables induce functions on $\Gamma$, the Dirac observables induce functions on $\Delta$.

\subsection{Interpretation}

Regarded as an action functional on the spacetime manifold $\mathcal{M}$, the covariant canonical action is equivalent to the Hilbert action coupled to causal reference systems ( $\mathbf{T}, \mathbf{X})$. Although the presence of these systems does not preclude the conventional interpretation of vacuum gravity based upon Hilbert action, it does imply that an additional postulate is necessary if this interpretation is to be recovered within the framework of the extended action. More precisely, the covariant canonical formalism accepts two different interpretations, depending on whether physical importance is ascribed to the entire set $\Gamma$ or solely to its subset $\Delta \subset \Gamma$. 
The second option amounts to the requirement, due to Bergmann [7] and Dirac [8], that all first-class constraints generate gauge transformations. According to this position, spacetime diffeomorphisms and deformations of the mappings $\mathbf{T}$ and $\mathbf{X}$ have no measurable consequences. The mappings $\mathbf{T}$ and $\mathbf{X}$ are deemed unimportant, and the physical observables coincide with the Dirac observables which are independent of these mappings. Since the Dirac observables do not evolve in time, the problem of evolution resurfaces in its standard form, as discussed in the literature [41]-[48]. In this case, the recovery of the representations of Diff $\mathcal{M}$ in the phase space of the covariant canonical action is devoid of physical significance.

Needless to say, prominence is given to the first option. According to this position, the set $\Delta$ does not exhaust the observable aspects of the theory. Significance is attributed to the entire set $\Gamma$, and the selection of the mappings $\mathbf{T}$ and $\mathbf{X}$ as additional variables advocates a specific physical proposition. This concerns the issue of what constitutes a physical spacetime in vacuum gravity; a long-standing issue that goes back to the founders of general relativity: Hilbert formalised the notion that the reference system in general relativity should be visualised as a fluid which carries clocks that keep a causal time [49], and Einstein used a similar idealisation in his book [50]. Stachel analysed the issue of observability in general relativity in Ref. [51, and Rovelli introduced the so-called localised and non-localised points of view in Ref. [52].

The concept of the reference fluid is realised in a mathematically precise way by the mappings $\mathbf{T}$ and $\mathbf{X}$. These mappings bridge the gap between observers and the system under observation in the absence of a physical process of measurement. Observers are assumed not to influence the gravitational system under observation. Although their trajectories have to be timelike, they do not form part of the physical system in the strict sense. Accordingly, the interaction between the mappings $\mathbf{T}, \mathbf{X}$ and the metric $\mathbf{G}$ is extremely tenuous. There is just enough interaction to distinguish between the points of $\mathcal{M}$, but not enough to disturb the geometry. This is captured by the vanishing energy-momentum of the fields $\mathbf{T}$ and $\mathbf{X}$ and the subsequent preservation of the vacuum constraints in the canonical theory.

Regarding determinism, initial data do not uniquely determine the evolution derived from the covariant canonical action, even after the orbits of Diff $\mathcal{M}$ have been eliminated. There is still freedom remaining in the theory due to the arbitrariness of the foliation. However, this does not mean that the gravitational system under observation has more freedom to evolve than it had before; i.e., when it was described by Hilbert's action. The freedom captured by the extended set $\Gamma$ only refers to the possibilities of observation associated with a given physical state $\delta \in \Delta$. As we shall see later, there is a whole set of states $\{\gamma\}$ in $\Gamma$ associated with each physical state $\delta \in \Delta$, all of which are Diff $\mathcal{M}$-invariant but foliation-dependent.

Provided that the set $\Gamma$ is considered meaningful, each such state $\gamma$ in the class $\{\gamma\}$ is accepted as a distinct measurable state of the physical state $\delta$. The underlying assumption is that distinct measurements of a given physical situation remain distinct even in the limit where the physical interaction between the observers and the gravitational system becomes negligible. In contrast, this kind of observability is rejected in the formulation based on Hilbert's action. The focus 
is placed there on the physical aspects of vacuum gravity in the strict sense, and hence only on the gravitational field. Indicative of this is the absence from that action functional of any variables representing systems of reference. Instead, this concept is relegated to the manifold charts or to external, auxiliary, elements of the theory.

Systems of reference appear in the conventional framework as a useful approximation, or even a necessary inconsistency, which is eliminated from the physical interpretation of the theory. Observers must have energy and momentum; otherwise, they cannot be considered as being part of the system. Non-dynamical is interpreted as physically unimportant, a fact which is declared by the bijective correspondence between the sets $\Gamma$ and $\Delta$. In particular, the metric field $\mathbf{G}$ at a given spacetime point is not observable; at least, not according to the physical premises of the theory as these follow from the selection of $\mathbf{G}$ as the sole variable in Hilbert's action.

These premises change after the mappings $\mathbf{T}$ and $\mathbf{X}$ are adjoined to the Hilbert action as additional variables. The emphasis is now placed on the admission of arbitrary reference systems which provides the empirical basis of Einstein's theory. These systems are treated in the same way as the metric field is, and the set $\Gamma$ is extended. Spacetime points are individuated by the presence of both the metric $\mathbf{G}$ and the fields $\mathbf{T}$ and $\mathbf{X}$, and the interactions $g, N, N^{i}$ between these fields are Diff $\mathcal{M}$-invariant and hence measurable. Within the extended phase space of the covariant canonical action, the Diff $\mathcal{M}$-induced first-class constraints generate gauge transformations, but the deformations of the mappings $\mathbf{T}$ and $\mathbf{X}$, despite being first-class, generate measurable changes of the time foliation and the reference frame. The aspects of the problem of time that touch on the classical theory are in this way overcome.

\subsection{Presentation}

The presentation is organised as follows. Section 2 summarises the relevant aspects of canonical general relativity, with particular emphasis being placed on the set $\Gamma$ of Diff $\mathcal{M}$-classes of solutions and the set $\Delta$ of first-class orbits. Section 3 illustrates the extension of the Hilbert action by non-dynamical variables and explains the subtleties of this procedure. This sets the stage for the main technical part of the paper, which begins in section 4 . There, the extended action is introduced in its spacetime form, and the corresponding canonical theory is derived. Section 5 investigates the set of solutions and the sets $\Gamma$ and $\Delta$ of the covariant canonical action and compares them with the corresponding sets of the Hilbert action. Section 6 describes the symmetries induced on the solutions of the field equations by the diffeomorphisms of $\mathcal{M}$ and the deformations of the mappings $\mathbf{T}$ and $\mathbf{X}$. Section 7 is concerned with the extended equal-time phase space $\mathcal{P}$, the action of symmetries on the instantaneous data, and their representations by symplectic diffeomorphisms of $\mathcal{P}$. Finally, section 8 considers the spacetime observables and their dynamical evolution, and discusses some conceptual aspects of the problem of time that are elucidated by the proposed formalism. 


\section{Background}

This section contains a summary of those aspects of general relativity that are pertinent to this paper. It is a collection of standard results. Conventions and terminology are introduced, and simplifying assumptions are made when necessary.

\subsection{The set $\Gamma$ of Diff $\mathcal{M}$-classes of solutions}

The action principle for general relativity postulates a four-dimensional background manifold $\mathcal{M}$ and an action functional $S[\boldsymbol{\Psi}]$ on $\mathcal{M}$. The variables $\boldsymbol{\Psi}$ include a metric field $\mathbf{G}$ and possibly other geometrical objects. The set of all kinematically admissible configurations of $\boldsymbol{\Psi}$ will be denoted by Virt $\mathcal{M}$ and referred to as the set of virtual fields. For example, in vacuum gravity, $\boldsymbol{\Psi}$ consists only of the metric field $\mathbf{G}$, and the set Virt $\mathcal{M}$ becomes the set Riem $\mathcal{M}$ of all pseudo-Riemannian metrics on $\mathcal{M}$. Each manifold

$$
\mathcal{M}_{\Psi}:=(\mathcal{M}, \Psi)
$$

associated with a configuration $\Psi \in \operatorname{Virt} \mathcal{M}$, will be referred to as a virtual manifold. After the variation of the action functional, the set $\operatorname{Sol} \mathcal{M} \subset \operatorname{Virt} \mathcal{M}$ of solutions consists of all $\Psi$ in Virt $\mathcal{M}$ that satisfy Einstein's equations. If $\boldsymbol{\Psi}$ belongs to $\operatorname{Sol} \mathcal{M}, \mathcal{M}_{\Psi}$ will be called a solution manifold.

There are as many distinct solution manifolds as there are distinct $\boldsymbol{\Psi} \in \operatorname{Sol} \mathcal{M}$. However, not all of them represent physically distinct states of the system. If there exists a diffeomorphism $D: \mathcal{M} \rightarrow \mathcal{M}$ such that

$$
\boldsymbol{\Psi}_{2}=D_{*} \boldsymbol{\Psi}_{1}
$$

where $D_{*}$ is the push-forward mapping, the solution manifolds $\mathcal{M}_{\boldsymbol{\Psi}_{1}}$ and $\mathcal{M}_{\boldsymbol{\Psi}_{2}}$ are considered equivalent; i.e., representations of the same physical state. For asymptotically flat spacetimes, the group Diff $\mathcal{M}$ has to be restricted so that it includes only those diffeomorphisms that act trivially at 'spatial infinity'. However, for simplicity, $\mathcal{M}$ will be assumed spatially compact in this paper.

The above considerations imply that the solution manifolds are divided into equivalence classes. Each equivalence class of solutions in $\operatorname{Sol} \mathcal{M}$ is identified with an element $\gamma$ of the quotient set

$$
\Gamma:=\operatorname{Sol} \mathcal{M} / o
$$

where $o$ denotes the orbits of Diff $\mathcal{M}$ in $\operatorname{Sol} \mathcal{M}$. The set $\Gamma$ will be referred to as the set of Diff $\mathcal{M}$-classes of solutions. The points of the background manifold $\mathcal{M}$ get entangled in $\Gamma$ with the solutions $\Psi$. This underlines the position that the points of the background $\mathcal{M}$ are physically indistinguishable in the absence of fields. In other words, the set $\Gamma$ captures the diffeomorphism invariance and background independence of general relativity. These two properties will be jointly referred to as general covariance. 


\subsection{The time, space, and foliation mappings}

The manifold $\mathcal{M}$ is assumed to be globally hyperbolic in order that Cauchy surfaces exist. By a theorem of Geroch [53], $\mathcal{M}$ has the topology $\Sigma \times \mathbb{R}$. Following Ref. [1], the elements $y$ of $\mathcal{M}$ will be called events, the elements $x$ of $\Sigma$ will be called points, and the elements $t$ of $\mathbb{R}$ will be called moments. Their coordinate representations are respectively $y^{\alpha}, x^{i}$ and $t$. A global time mapping $\mathbf{T}$ is a function

$$
\mathbf{T}: \mathcal{M} \rightarrow \mathbb{R} \text { by } y \in \mathcal{M} \mapsto t=\mathbf{T}(y) \in \mathbb{R}
$$

from $\mathcal{M}$ to the real numbers. For each solution manifold $\mathcal{M}_{\boldsymbol{\Psi}}$, the gradient $\mathbf{T}_{, \alpha}$ of $\mathbf{T}$ has to be timelike with respect to the metric field $G \in \mathbf{\Psi}$; i.e., $\mathbf{G}^{\alpha \beta} \mathbf{T}_{, \alpha} \mathbf{T}_{, \beta}<0$. This means that $\mathbf{T}$ must depend on $\boldsymbol{\Psi}$. Each such mapping $\mathbf{T}$ associates a spacelike hypersurface $\Sigma_{(t)}^{\mathbf{T}}$ in $\mathcal{M}$ with a moment $t$ of $\mathbb{R}$ :

$$
\Sigma_{(t)}^{\mathbf{T}}=\{y \in \mathcal{M}: \mathbf{T}(y)=t \in \mathbb{R}\} .
$$

Such a hypersurface will be called an instant and their collection

$$
\Sigma^{\mathbf{T}}:=\left\{\Sigma_{(t)}^{\mathbf{T}}: t \in \mathbb{R}\right\}
$$

a time foliation of $\mathcal{M}$. The time map $\mathbf{T}$ is required to respect the orientation of $\mathcal{M}$ : if $t_{1}<t_{2}$, the instant $\Sigma_{\left(t_{2}\right)}^{\mathbf{T}}$ has to lie in the future of $\Sigma_{\left(t_{1}\right)}^{\mathbf{T}}$ in $\mathcal{M}$.

The space mapping $\mathbf{X}$ accompanying $\mathbf{T}$ is a mapping

$$
\mathbf{X}: \mathcal{M} \rightarrow \Sigma \text { by } y \in \mathcal{M} \mapsto x=\mathbf{X}(y) \in \Sigma
$$

from $\mathcal{M}$ to a three-dimensional manifold $\Sigma$, which is assumed compact. The local coordinate representation of $\mathbf{X}$ is $\mathbf{X}^{i}$. The gradients $\mathbf{X}_{, \alpha}^{i}$ have to be spacelike; i.e., for each $i$, we must have that $\mathbf{G}^{\alpha \beta} \mathbf{X}_{, \alpha}^{i} \mathbf{X}_{, \beta}^{i}>0$. Therefore, $\mathbf{X}$ has to depend on $\boldsymbol{\Psi}$ as well. Each such mapping $\mathbf{X}$ associates a timelike worldline $C_{(x)}^{\mathbf{X}}$ in $\mathcal{M}$ with a point $x$ of $\Sigma$ :

$$
C_{(x)}^{\mathbf{X}}=\{y \in \mathcal{M}: \mathbf{X}(y)=x \in \Sigma\} .
$$

This will be called a reference worldline and their collection

$$
C^{\mathbf{X}}=\left\{C_{(x)}^{\mathbf{X}}: x \in \Sigma\right\}
$$

a reference frame.

The Cartesian product

$$
\begin{aligned}
\mathbf{T} \times \mathbf{X}: \mathcal{M} & \rightarrow \mathbb{R} \times \Sigma \text { by } \\
y \in \mathcal{M} & \mapsto(t=\mathbf{T}(y) \in \mathbb{R}, x=X(y) \in \Sigma)
\end{aligned}
$$

associates the event $y \in \mathcal{M}$ with the moment $t \in \mathbb{R}$ and the point $x \in \Sigma$. Its inverse mapping

$$
Y: \mathbb{R} \times \Sigma \rightarrow \mathcal{M} \text { by }(t \in \mathbb{R}, x \in \Sigma) \mapsto y=Y(t, x) \in \mathcal{M}
$$


will be called the foliation mapping. It may be viewed as a one-parameter family $Y_{(t)}, t \in \mathbb{R}$ of embeddings

$$
Y_{(t)}: \Sigma \rightarrow \mathcal{M} \text { by } x \in \Sigma \mapsto Y_{(t)}(x):=Y(t, x) \in \mathcal{M}
$$

of $\Sigma$ into $\mathcal{M}$, whose images $\Sigma_{(t)}^{\mathbf{T}}=Y_{(t)}(\Sigma)$ define the foliation $\Sigma^{\mathbf{T}}=\left\{\Sigma_{(t)}^{\mathbf{T}}, t \in \mathbb{R}\right\}$ of $\mathcal{M}$. It may also be viewed as a congruence $Y_{(x)}, x \in \Sigma$ of curves

$$
Y_{(x)}: \mathbb{R} \rightarrow \mathcal{M} \text { by } t \in \mathbb{R} \mapsto Y_{(x)}(t):=Y(t, x) \in \mathcal{M},
$$

whose images $C_{(x)}^{\mathbf{X}}=Y_{(x)}(\mathbb{R})$ define the reference frame $C^{\mathbf{X}}=\left\{C_{(x)}^{\mathbf{X}}, x \in \Sigma\right\}$ in $\mathcal{M}$. The foliation mapping $Y$ locates the event $y \in \mathcal{M}$ at which the instant $\Sigma_{(t)}^{\mathbf{T}}=Y_{(t)}(\Sigma)$ intersects the reference worldline $C_{(x)}^{\mathbf{X}}=Y_{(x)}(\mathbb{R})$.

\subsection{The transition from $\mathcal{M}$ to $\Sigma \times \mathbb{R}$}

Given an arbitrary configuration $\boldsymbol{\Psi}$ in $\operatorname{Virt} \mathcal{M}$, only a subset $\{(\mathbf{T}, \mathbf{X})\}$ of mappings will respect the light-cone structure induced on $\mathcal{M}$ by the metric $\mathbf{G}$ in $\boldsymbol{\Psi}$. The requirements that $\Sigma^{\mathbf{T}}$ be spacelike with respect to $\mathbf{G}$ and $C^{\mathbf{X}}$ be timelike with respect to $\mathbf{G}$ imposes some restrictions on the induced fields $\psi:=Y^{*} \Psi$ on $\Sigma \times \mathbb{R}$. For example, the pullback metric $g_{(t)}:=Y_{(t)}^{*} \mathbf{G}$ induced on $\Sigma$ by $\mathbf{G}$ and a given embedding $Y_{(t)}$ has to be positive definite for all $t$. These restrictions on the fields $\psi$ on $\Sigma \times \mathbb{R}$ will be called the compatibility conditions, and the corresponding mappings $\{(\mathbf{T}, \mathbf{X})\}$ will be called compatible with the configuration $\Psi$ on $\mathcal{M}$.

Following the presentation of the Dirac-ADM approach as given in Ref. [41], the action is viewed as a functional of a reference configuration $\boldsymbol{\Psi}_{o}$ in $\operatorname{Virt} \mathcal{M}$ and is pulled back from $\mathcal{M}$ to $\Sigma \times \mathbb{R}$ by a reference mapping $Y_{o}$ whose associated time foliation $\Sigma^{\mathbf{T}_{o}}$ and reference frame $C^{\mathbf{X}_{o}}$ are compatible with $\mathbf{G}_{o} \in \boldsymbol{\Psi}_{o}$. When the action functional $S\left[\boldsymbol{\Psi}_{o}\right]$ on $\mathcal{M}$ is pulled back to $\Sigma \times \mathbb{R}$, the mapping $Y_{o}$ drops out of the resulting functional, and the field equations are expressed exclusively in terms of the induced configuration $\psi_{o}$ on $\Sigma \times \mathbb{R}$. This important feature enables the theory to be expressed in the new context without the connecting mappings $\mathbf{T}, \mathbf{X}$ and $Y$ taking any further part in its formulation. In particular, the compatibility conditions for the induced fields $\psi_{o}$ are turned into definitions for the set $\operatorname{Virt}(\Sigma \times \mathbb{R})$ of induced virtual fields $\psi$ on $\Sigma \times \mathbb{R}$. For example, the definition for the symmetric tensor $g_{(t)}$ on $\Sigma$ is that it has to be positive definite for all $t \in \mathbb{R}$. In this way, the action functional $S[\psi]$ is subsequently varied independently of any link with $\mathcal{M}$.

Assuming that four of the induced fields $\psi$ on $\Sigma \times \mathbb{R}$ become Lagrange multipliers in the canonical theory, the induced fields can be expressed in the familiar form $\psi=\left(q, N, N^{i}\right)$. The lapse $N$ and the shift $N^{i}$ are respectively scalar and vector fields on $\Sigma$. They are both scalar densities of weight one on $\mathbb{R}$. The dynamical fields $q$, which include the induced metric $g$, may be chosen as tensors on $\Sigma$ and as scalars on $\mathbb{R}$. Momenta $p$ conjugate to $q$ are introduced. These are tensor densities of weight one on $\Sigma$ and scalars on $\mathbb{R}$. The Legendre transformation 
brings the Lagrangian action $S\left[q, N, N^{i}\right]$ on $\Sigma \times \mathbb{R}$ into the canonical form

$$
S\left[q, p ; N, N^{i}\right]=\int d t d^{3} x\left(p \dot{q}-N H-N^{i} H_{i}\right)+\int d t d^{3} x \dot{B}+\int d t d^{3} x B_{, i}^{i} .
$$

The functionals $H(q, p)$ and $H_{i}(q, p)$ are the super-Hamiltonian and super-momenta of the system. The boundary contributions $B$ and $B^{i}$ depend on $N, N^{i}, q$ and $p$. Since $\Sigma$ has been assumed compact, the spatial divergence vanishes.

The field equations on $\Sigma \times \mathbb{R}$ are equivalent to Einstein's equations on $\mathcal{M}$ in the following sense: Given any solution $\boldsymbol{\Psi}$ of Einstein's equations and any pair $(\mathbf{T}, \mathbf{X})$ that is compatible with $\mathbf{G} \in \boldsymbol{\Psi}$, the induced fields $\psi$ satisfy the compatibility conditions and the field equations on $\Sigma \times \mathbb{R}$. Conversely, given any foliation mapping $Y$ and any configuration $\psi$ in $\operatorname{Virt}(\mathbb{R} \times \Sigma)$ that satisfies the field equations on $\Sigma \times \mathbb{R}$, the reconstructed configuration $\Psi$ satisfies Einstein's equations on $\mathcal{M}$ and is such that the Cartesian product mapping $\mathbf{T} \times \mathbf{X}$ inverse to $Y$ is compatible with $\mathbf{G} \in \Psi$.

The following results are also relevant, stated, for example, by Hájíček and Kijowski [54]: Given a solution manifold $\mathcal{M}_{\boldsymbol{\Psi}}$ and any compatible mapping $\mathbf{T} \times \mathbf{X}$, each hypersurface $\Sigma_{\left(t_{o}\right)}^{\mathbf{T}} \subset$ $\mathcal{M}_{\Psi}, t_{o} \in \mathbb{R}$, is an admissible Cauchy surface; i.e., a possible initial manifold for Einstein's equations. Let the initial datum induced on $\Sigma$ by $\boldsymbol{\Psi}$ and the embedding $Y_{\left(t_{o}\right)}: \Sigma \rightarrow \Sigma_{\left(t_{o}\right)}^{\mathbf{T}}$ be $(q, p)$. Then, this datum $(q, p)$ determines a unique class $\{\boldsymbol{\Psi}\}$ of Diff $\mathcal{M}$-related solutions in Sol $\mathcal{M}$ in the following sense: For any choice of lapse $N$ and shift $N^{i}$, and for any choice of foliation mapping $Y$, the maximal dynamical development of $(q, p)$ reconstructs one of the solution manifolds in the class $\{\boldsymbol{\Psi}\}$.

\subsection{The reduced phase space $\Delta$ and the physical observables}

The phase space $\mathcal{P}$ may be defined as the cotangent bundle over the set of the fields $q$ on $\Sigma$ equipped with a weak symplectic form. Initial data appropriate for Einstein's equations lie on the constraint surface $\mathcal{C} \subset \mathcal{P}$ determined by $H(q, p)=0$ and $H^{i}(q, p)=0$. These data do not all determine physically distinct states of the system. All points $(q, p)$ that lie in an orbit of the Hamiltonian vector field generated by the functional

$$
H\left[q, p ; N, N^{i}\right]=\int d^{3} x\left(N H-N^{i} H_{i}\right)
$$

determine the same physical state. Specifically, for each choice of the smearing functions $N$ and $N^{i}$, the functional (15) generates a one-parameter family of canonical transformations of $\mathcal{P}$ that develops a given datum $(q, p)$ into a curve of data in $\mathcal{C}$. The subset of $\mathcal{C}$ that can be reached from this point $(q, p) \in \mathcal{C}$ by the action of (15) via all possible smearing functions defines the orbit $o_{(q, p)}$ in $\mathcal{C}$. Since the datum $(q, p)$ determines a unique Diff $\mathcal{M}$-class $\{\boldsymbol{\Psi}\}$ of solutions in SolM, it follows that each datum $\left(q^{\prime}, p^{\prime}\right)$ in the orbit $o_{(q, p)}$ determines the same Diff $\mathcal{M}$-class $\{\boldsymbol{\Psi}\}$ of solutions as $(q, p)$. It is in this sense that it is can be claimed that the Hamiltonian (15) "generates" spacetime diffeomorphisms. 
The quotient set $\Delta$,

$$
\Delta:=\mathcal{C} / o,
$$

where $o$ denotes the orbits generated on $\mathcal{C}$ by (15), becomes the reduced phase space of the theory once the symplectic form on $\mathcal{P}$ is pulled-back to $\Delta$. Each element $\delta \in \Delta$ represents a Diff $\mathcal{M}$-class of solutions in $\operatorname{Sol} \mathcal{M}$. The correspondence between the sets $\Gamma$ and $\Delta$ is therefore one-to-one, and the counting of physically distinct states according to $\Gamma$ is recovered within the canonical theory.

Regarding the physical observables, these are the Dirac observables. Specifically, two functions $F_{1}(q, p)$ and $F_{2}(q, p)$ are considered equivalent on $\mathcal{P}$ if their values are equal on the constraint surface $\mathcal{C}$,

$$
\left.F_{1}(q, p)\right|_{\mathcal{C}}=\left.F_{2}(q, p)\right|_{\mathcal{C}} .
$$

Each such equivalence class of functions, represented by $F$, defines a Dirac observable if, for all choices of $N$ and $N^{i}, F$ commutes on $\mathcal{C}$ with the Hamiltonian:

$$
\left.\left\{F(q, p), H\left[q, p ; N, N^{i}\right]\right\}\right|_{\mathcal{C}}=0 .
$$

The Dirac observables $F$ on $\mathcal{P}$ can be projected via (16) to functionals on $\Delta$, as explained, for example, by Fischer and Marsden [55]; they may also be projected to functionals on $\Gamma$, as discussed by Hájíček [10]. In either case, these observables remain invariant under the dynamical evolution generated by the Hamiltonian. Since nothing physical actually evolves, this evolution cannot be regarded as being measurable.

\section{Extending and reducing the action}

This section is concerned with the main concept behind the present approach. Namely, it considers the procedure of incorporating non-dynamical variables into the variational principle of a Diff $\mathcal{M}$-invariant action. It investigates the effect of this procedure on the sets $\Gamma$ and $\Delta$ of the resulting action and the consequences this has for the emerging spacetime. In some respects, this topic is reminiscent of the issues raised by Kretschmann [58], Cartan [59] and Fock [60], when they discussed the extent to which Einstein's general theory obeys a relativity principle. Connections may also be recognised with the issues investigated by Anderson [61, Kuchař [62] and more recently by Sorkin [63]. However, no attempt will be made to relate the contents of this section to one of these viewpoints.

\subsection{The method of extension}

In order to illustrate the extension of the Hilbert action without the complications arising from the compatibility conditions between $\mathbf{T}, \mathbf{X}$ and $\mathbf{G}$, it is best to put aside these conditions 
for the moment, and incorporate them in the next section. Thus, assuming that there is no relationship between $\mathbf{T}, \mathbf{X}$ and $\mathbf{G}$, one can add these mappings to the Hilbert action,

$$
S[\mathbf{G}]=\int_{\mathcal{M}} \mathbf{d}^{4} \mathbf{y} \sqrt{-\operatorname{det} \mathbf{G}} \mathbf{R}[\mathbf{G}],
$$

in a technically trivial way, without spoiling the general covariance or the dynamical content of the field equations. There are two stages in this procedure, which follows Ref. [1]: First, the set $\operatorname{Virt} \mathcal{M}=\operatorname{Riem} \mathcal{M}$ of virtual fields of (19) is extended by the set Maps $\mathcal{M}$ of the time and space mappings, $\mathbf{T}: \mathcal{M} \rightarrow \mathbb{R}$ and $\mathbf{X}: \mathcal{M} \rightarrow \Sigma$, and becomes the product set

$$
\operatorname{Virt} \mathcal{M}:=\operatorname{Maps} \mathcal{M} \times \operatorname{Riem} \mathcal{M}
$$

Second, the action is viewed as a functional of all the variables $\mathbf{T}, \mathbf{X}$ and $\mathbf{G}$,

$$
S[\mathbf{T}, \mathbf{X}, \mathbf{G}]=\int_{\mathcal{M}} \mathbf{d}^{4} \mathbf{y} \sqrt{-\operatorname{det} \mathbf{G}} \mathbf{R}[\mathbf{G}],
$$

without $\mathbf{T}$ and $\mathbf{X}$ actually appearing on the right hand side of (21).

In spite of the simplicity of this procedure, the distinction between actions (19) and (21) is grounded on their sets $\Gamma$. In particular, the set Virt $\mathcal{M}$ of virtual fields of (21) is larger than the corresponding set of (19). In addition, the variation of $\mathbf{T}$ and $\mathbf{X}$ in (21) yields generallycovariant field equations - in fact, identities - that leave $\mathbf{T}$ and $\mathbf{X}$ arbitrary. Therefore, virtual fields and solutions for $\mathbf{T}$ and $\mathbf{X}$ are one and the same. If a particular metric $\mathbf{G}$ solves Einstein's vacuum equations, i.e., if $\mathbf{G}$ belongs to the set $\operatorname{Sol}^{\mathrm{Hil}} \mathcal{M}$ of $(\underline{19})$, then any pair $\mathbf{\Psi}=(\mathbf{T}, \mathbf{X}, \mathbf{G})$, where $(\mathbf{T}, \mathbf{X}) \in \operatorname{Maps} \mathcal{M}$, belongs to the extended set of solutions $\operatorname{Maps} \mathcal{M} \times \operatorname{Sol}^{\mathrm{Hil}} \mathcal{M}$ of (21). As a result, the set $\Gamma$ of Diff $\mathcal{M}$-classes of solutions of (21) is larger than that of (19).

Furthermore, the fact that the variables $\mathbf{T}$ and $\mathbf{X}$ are absent from the right hand side of (21) cannot be used as an argument for rejecting (21) as a genuine action. The reason is that a different choice of variables in the set Virt $\mathcal{M}$ of (21) can make the right hand side of (21) depend explicitly on all these variables. For example, one may choose the ADM chart on Virt $\mathcal{M}$ consisting of the variables $\mathbf{T}, \mathbf{X}, \mathbf{N}, \mathbf{N}^{i}$ and $\mathbf{g}_{i j}$, where the boldface lapse $\mathbf{N}$, shift $\mathbf{N}^{i}$ and spatial metric $\mathbf{g}_{i j}$ are viewed as scalar functions on $\mathcal{M}$. In this case, action (21) becomes

$$
S\left[\mathbf{T}, \mathbf{X}, \mathbf{N}, \mathbf{N}^{i}, \mathbf{g}_{i j}\right]=\int_{\mathcal{M}} d^{4} y\left|\frac{\partial(\mathbf{T}, \mathbf{X})}{\partial y^{\alpha}}\right| \mathbf{N} \sqrt{\operatorname{det} \mathbf{g}}\left(\mathbf{k}_{i j} \mathbf{k}^{i j}-\left(\mathbf{k}_{i}^{i}\right)^{2}+R[\mathbf{g}]\right) .
$$

The boldface extrinsic curvature $\mathbf{k}_{i j}$ and the spatial curvature scalar $R[\mathbf{g}]$ are also viewed as scalar functions on $\mathcal{M}$. The Jacobian $\left|\frac{\partial(\mathbf{T}, \mathbf{X})}{\partial y^{\alpha}}\right|$ in (22) depends on the mappings $\mathbf{T}$ and $\mathbf{X}$ and, therefore, all the elements of the set Virt $\mathcal{M}$ are present in this version of the action. In particular, the variation of (22) produces equations that are all generally-covariant and nontrivial. Thus, despite appearances, actions (19) and (21) are distinct in agreement with their distinct sets $\Gamma$, while actions (21) and (22) are just equivalent.

If now physical significance is attributed to the set $\Gamma$, the process of extension of the Hilbert action by non-dynamical variables has observable consequences. And, dragging this argument 
a little further, the principle of general covariance cannot, by itself, stop such a proliferation of observables. For example, a variety of spacetime fields may be added to the set Virt $\mathcal{M}$ of (19), without any of them appearing in the action. All these fields will remain undetermined by the variational principle, and this will produce yet another set $\Gamma$ of observables, while leaving the general covariance of the formalism intact. Under these circumstances, the immediate need arises for establishing a criterion that can decide on the appropriateness of the selected variables. Given that the principle of general covariance is not such a criterion, one has to resort to the interpretation of the chosen variables, and require that this interpretation be consistent with observation; i.e., that any predicted observables be actually observed. Seen in this light, the formalism based on (21) is clearly inappropriate, because the mappings $(\mathbf{T}, \mathbf{X})$ are entirely independent of $\mathbf{G}$, while reference systems are causal.

\subsection{Reduction via Dirac's requirement}

Before modifying (21) by introducing compatibility conditions between $\mathbf{T}, \mathbf{X}$ and $\mathbf{G}$, it is worth considering the reverse procedure; namely, the reduction from (21) to the original action (19). Although these formalisms are distinct according to their sets $\Gamma$, it is also true that they look similar. This similarity is established by the Bergmann-Dirac analysis of the first-class constraints. More precisely, while the differences between (19) and (21) are expressed by the set $\Gamma$ of Diff $\mathcal{M}$-classes of solutions, their similarities are expressed by the set $\Delta$ of first-class orbits.

Actions (19) and (21) yield sets $\Delta$ that are isomorphic. In the case of (19), the correspondence between the sets $\Gamma$ and $\Delta$ is bijective, allowing the Hamiltonian and momentum constraints to be associated with Diff $\mathcal{M}$. In the case of (21), any canonical analysis that incorporates the non-dynamical variables $\mathbf{T}$ and $\mathbf{X}$ in the phase space will yield eight first-class constraints, four too many to be associated with Diff $\mathcal{M}$. The remaining four first-class constraints reflect the fact that the mappings $\mathbf{T}$ and $\mathbf{X}$ are left undetermined by the variational principle. These extra constraints imply that the set $\Delta$ of (21) is a subset of its set $\Gamma$, and isomorphic to the set $\Delta$ of (19).

Now, if the point of view is followed that the observable aspects of a generally-covariant theory are associated solely with its set $\Delta$, then some of the physical premises of (21), according to its set $\Gamma$, have to be invalidated. This reduction from the physical premises of (21) to those of (19) can be achieved by postulating, following Bergmann and Dirac, that all eight firstclass constraints in the phase space of (21) generate gauge transformations. This is sometimes referred to as Dirac's conjecture - analysed in detail in Refs. [8], [56] and [57] - although it may be regarded as a physical requirement.

Imposing this requirement on the formalism defined by (21) has the following consequences: If all first-class constraints generate gauge transformations, then taking the quotient of the set

of solutions of (21) by the orbits of Diff $\mathcal{M}$ is not sufficient to eliminate all gauge freedom. It is necessary to take the quotient of the resulting set $\Gamma$ by the orbits in $\Gamma$ generated by arbitrary 
changes of the fields $\mathbf{T}$ and $\mathbf{X}$. These changes are symmetries of the field equations derived from (21). The fact that these symmetries are physically unimportant can be deduced from the analysis of the first-class constraints and the transformations these generate in the phase space. The quotient of $\Gamma$ by the orbits generated by these symmetries yields a set that is isomorphic to the set $\Delta$.

\section{The extended action}

The ability to reduce the physical content of the extended formalism to that of the conventional formalism by making use of Dirac's requirement means that it is not necessary to commit to a particular interpretation. Both options for interpreting canonical vacuum gravity are incorporated in the extended action. Thus, in this section, the compatibility conditions between $\mathbf{T}, \mathbf{X}$ and $\mathbf{G}$ are added to action (21), and the covariant canonical formalism is derived. By necessity, the following four sections are technical. However, the conceptual characteristics of the formalism are discussed again in the last section.

\subsection{The Lagrangian on $\mathcal{M}$}

Given coordinates $y^{\alpha}$ on $\mathcal{M}, x^{i}$ on $\Sigma$, and $t$ on $\mathbb{R}$, the metric field $\mathbf{G}_{\alpha \beta}$ is pulled back to $\Sigma \times \mathbb{R}$ by the foliation mapping $Y$ to yield the induced metric $g_{i j}$, the shift vector $N^{i}$ and the lapse function $N$

$$
\begin{aligned}
\mathbf{G}_{\alpha \beta}(Y) Y_{, i}^{\alpha} Y_{, j}^{\beta} & :=g_{i j}, \\
\mathbf{G}_{\alpha \beta}(Y) Y_{, i}^{\alpha} Y_{, t}^{\beta} & :=g_{i j} N^{j}, \\
\mathbf{G}_{\alpha \beta}(Y) Y_{, t}^{\alpha} Y_{, t}^{\beta} & :=g_{i j} N^{i} N^{j}-N^{2} .
\end{aligned}
$$

Alternatively, $\mathbf{G}$ may be expanded in the $\mathbf{G}$-independent co-basis constructed from the mappings $\mathbf{T}$ and $\mathbf{X}$, according to

$$
\mathbf{G}_{\alpha \beta}=\left(\mathbf{g}_{i j} \mathbf{N}^{i} \mathbf{N}^{j}-\mathbf{N}^{2}\right) \mathbf{T}_{, \alpha} \mathbf{T}_{, \beta}+\mathbf{g}_{i j} \mathbf{N}^{j}\left(\mathbf{T}_{, \alpha} \mathbf{X}_{, \beta}^{i}+\mathbf{X}_{, \alpha}^{i} \mathbf{T}_{, \beta}\right)+\left(\mathbf{g}_{i j} \mathbf{X}_{, \alpha}^{i} \mathbf{X}_{, \beta}^{j}\right),
$$

where the boldface lapse, shift and spatial-metric are viewed as fields on $\mathcal{M}$ :

$$
\begin{aligned}
\mathbf{g}_{i j} & :=g_{i j}(\mathbf{T}, \mathbf{X}), \\
\mathbf{N} & :=N(\mathbf{T}, \mathbf{X}), \\
\mathbf{N}^{i} & :=N^{i}(\mathbf{T}, \mathbf{X}) .
\end{aligned}
$$

Equation (26), which provides the link between the spacetime fields and the fields on $\mathbb{R} \times \Sigma$, is adjoined to the extended action (21) by Lagrange multipliers. The independent variables in the resulting action

$$
S\left[\mathbf{G}, \mathbf{T}, \mathbf{X}, N, N^{i}, g, \mathbf{M}\right]=\int d^{4} X \sqrt{-\operatorname{det} \mathbf{G}}\left(R[\mathbf{G}]+\mathbf{M}^{\alpha \beta} \mathbf{C}_{\alpha \beta}\right)
$$


are chosen to be the metric field $\mathbf{G}$, the mappings $\mathbf{T}$ and $\mathbf{X}$, the induced fields $N, N^{i}$ and $g$ on $\Sigma \times \mathbb{R}$ and finally the symmetric tensor density multipliers $\mathbf{M}$. The tensor field

$$
C_{\alpha \beta}:=\mathbf{G}_{\alpha \beta}-\left(\mathbf{g}_{\mathbf{i j}} \mathbf{N}^{\mathbf{i}} \mathbf{N}^{\mathbf{j}}-\mathbf{N}^{\mathbf{2}}\right) \mathbf{T}_{, \alpha} \mathbf{T}_{, \beta}-\mathbf{g}_{\mathbf{i j}} \mathbf{N}^{\mathbf{j}}\left(\mathbf{T}_{, \alpha} \mathbf{X}_{, \beta}^{\mathbf{i}}+\mathbf{X}_{, \alpha}^{\mathbf{i}} \mathbf{T}_{, \beta}\right)-\mathbf{g}_{\mathbf{i j}} \mathbf{X}_{, \alpha}^{\mathbf{i}} \mathbf{X}_{, \beta}^{\mathbf{j}},
$$

is just a re-arrangement of (26) , where the boldface lapse, shift and induced metric are viewed as functionals of the independent variables $N, N^{i}, g_{i j}, \mathbf{T}$ and $\mathbf{X}$ via (27)-(29). The space of virtual fields of (30) incorporates the appropriate definitions for the induced fields $\left(g, N, N^{i}\right)$ on $\Sigma \times \mathbb{R}$ that ensure compatibility between $\mathbf{T}, \mathbf{X}$ and $\mathbf{G}$ at the level of the solutions. On the other hand, the mappings $\mathbf{T}$ and $\mathbf{X}$ are unrestricted prior to variation. The manifest invariance of the action (30) under the diffeomorphisms of all manifolds $\mathcal{M}, \mathbb{R}$ and $\Sigma$ should be noted.

The dynamical content of the equations derived from (30) is equivalent to that of Einstein's equations for vacuum gravity. In particular, the variation of (30) with respect to the multipliers $\mathbf{M}$ yields the constraint (26), relating the spacetime fields with the fields on $\mathbb{R} \times \Sigma$. Since the compatibility conditions have been incorporated in the set Virt $\mathcal{M}$ of (30), constraint (26) ensures that the mappings $\mathbf{T}$ and $\mathbf{X}$ are compatible with $\mathbf{G}$ at the level of the solutions. The variation of (30) with respect to $N, N^{i}$ and $g$ implies that all projections of the multipliers $\mathbf{M}$, and hence $\mathbf{M}$ themselves, vanish:

$$
\begin{aligned}
\mathbf{M}^{\alpha \beta} \mathbf{T}_{, \alpha} \mathbf{T}_{, \beta} & =0 \\
\mathbf{M}^{\alpha \beta}\left(\mathbf{T}_{, \alpha} \mathbf{X}_{, \beta}^{i}+\mathbf{X}_{, \alpha}^{i} \mathbf{T}_{, \beta}\right) & =0 \\
\mathbf{M}^{\alpha \beta} \mathbf{X}_{, \alpha}^{i} \mathbf{X}_{, \beta}^{j} & =0 .
\end{aligned}
$$

Regarding the variation of $\mathbf{G}$, the contributions arising from the second term in (30) are proportional to the vanishing multipliers $\mathbf{M}$. Therefore, the vacuum Einstein equations are preserved:

$$
R^{\alpha \beta}[\mathbf{G}]-\frac{1}{2} R[\mathbf{G}] \mathbf{G}^{\alpha \beta}=0 .
$$

Finally, the variations of the time mapping $\mathbf{T}$ and the space mapping $\mathbf{X}$ yield equations that are valid by means of the remaining field equations, and hence provide no additional information.

\subsection{The Lagrangian on $\mathbb{R} \times \Sigma$}

Following [1], the transition from $\mathcal{M}$ to $\Sigma \times \mathbb{R}$ is viewed as a change of "coordinate chart" on the set of virtual fields of (30): The pair of mappings $(\mathbf{T}, \mathbf{X})$ is replaced by its inverse mapping $Y$, the multiplier $\mathbf{M}$ is replaced by its components $\left(M, M^{i}, M^{i j}\right)$ in the $\mathbf{G}$-independent basis on $\mathcal{M}$ constructed from $Y$,

$$
M Y_{, t}^{\alpha} Y_{, t}^{\beta}+\frac{1}{2} M^{i}\left(Y_{, t}^{\alpha} Y_{, i}^{\beta}+Y_{, i}^{\alpha} Y_{, t}^{\beta}\right)+M^{i j} Y_{, i}^{\alpha} Y_{, j}^{\beta}:=\mathbf{M}^{\alpha \beta}(Y)
$$

and the action (30) is pulled back to an equivalent Lagrangian action on $\Sigma \times \mathbb{R}$ :

$S\left[\mathbf{G}, Y, N, N^{i}, g, M, M^{i}, M^{i j}\right]=\int d^{3} x d t N \sqrt{\operatorname{det} g}\left(\kappa_{i j} \kappa^{i j}-\left(\kappa_{i}^{i}\right)^{2}+R[g]+M C+M^{i} C_{i}+M^{i j} C_{i j}\right)$. 
The constraints

$$
\begin{aligned}
C & :=\mathbf{G}_{\alpha \beta}(Y) Y_{, t}^{\alpha} Y_{, t}^{\beta}-g_{i j} N^{i} N^{j}+N^{2}, \\
C_{i} & :=\mathbf{G}_{\alpha \beta}(Y) Y_{, t}^{\alpha} Y_{, i}^{\beta}-g_{i j} N^{j} \\
C_{i j} & :=\mathbf{G}_{\alpha \beta}(Y) Y_{, i}^{\alpha} Y_{, j}^{\beta}-g_{i j}
\end{aligned}
$$

reproduce definitions (23)-(25),$\kappa$ is the extrinsic curvature

$$
\kappa_{i j}:=\frac{1}{2 N}\left(-\dot{g}_{i j}+N_{i ; j}+N_{j ; i}\right)
$$

and $R[g]$ is the curvature scalar. The constraints (38)-(40) have been already used to eliminate all other occurrences of $\mathbf{G}$ in the action functional; a procedure that is always permitted.

Now, in general, a set of variables can be eliminated completely from the formalism as long as the field equations obtained by varying these variables in the action functional determine the varied variables uniquely. The relevant information is preserved by turning these equations into definitions for the eliminated variables. In the case of (37), the simultaneous variation of the multipliers $\left(M, M^{i}, M^{i j}\right)$ and the metric field $\mathbf{G}$ implies that these multipliers vanish,

$$
M=0, \quad M^{i}=0, \quad M^{i}=0,
$$

and determines $\mathbf{G}$ uniquely in terms of the remaining variables according to (23) $-(25)$. A key property of this procedure is that, although it reduces the size of the set of virtual fields, it preserves the subset of solutions and consequently the set $\Gamma$ of Diff $\mathcal{M}$-classes of solutions. This follows from the uniqueness of its outcome; i.e., from the fact that the eliminated variables are expressed uniquely in terms of the variables that remain in the theory. In the particular case of (37), after the redundant variables $M, M^{i}, M^{i j}$ and $\mathbf{G}$ are eliminated, the mapping $Y$ drops out of the right hand side of the simplified action as well:

$$
S\left[Y, N, N^{i}, g\right]=\int d^{3} x d t N \sqrt{\operatorname{det} g}\left(\kappa_{i j} \kappa^{i j}-\left(\kappa_{i}^{i}\right)^{2}+R[g]\right) .
$$

However, it should not be forgotten that this mapping is still present in the set of virtual fields of (43), reflecting the fact that the set $\Gamma$ of (43) is larger than the corresponding set of the Hilbert action. As a confirmation of the consistency of this reasoning, $Y$ cannot be eliminated by means of its own variation in (43), since this variation yields an identity which is unable to determine $Y$ uniquely.

\subsection{The covariant canonical action}

Momenta conjugate to both $g$ and $Y$ need to be introduced. The Legendre transformation followed by the Dirac analysis of the primary constraints brings (43) into the first-class canonical form

$$
S\left[Y, P, q, p ; N, N^{i}, \Lambda\right]=\int d t d^{3} x\left(P_{\alpha} Y_{, t}^{\alpha}+p^{i j} q_{i j, t}-N H-N^{i} H_{i}-\Lambda^{\alpha} P_{\alpha}\right),
$$


where $\Lambda^{\alpha}, N$ and $N^{i}$ are Lagrange multipliers. In contrast to the mappings $\mathbf{T}$ and $\mathbf{X}$, which satisfy the compatibility constraint (31), the mapping $Y$ remains entirely arbitrary after the variation of (44). This is because, in this version of the extended action, the compatibility conditions are fully integrated into the set of virtual fields on $\mathbb{R} \times \Sigma$ and, hence, are transferred directly to the level of the solutions. In particular, since $g \in \operatorname{Virt} \mathcal{M}$ is positive definite, and $N \in \operatorname{Virt} \mathcal{M}$ is positive, any solution $(g, p)$ of (44) combined with any foliation mapping $Y$ reconstruct a spacetime metric $\mathbf{G}$ which is, by construction, compatible with the mappings $\mathbf{T}$ and $\mathbf{X}$ inverse to $Y$.

Thus, the variation of (44) yields the standard equations of the Dirac-ADM action,

$$
\begin{aligned}
g_{i j, t} & =\left\{g_{i j}, \int d^{3} x\left(N H+N^{m} H_{m}+\Lambda^{\beta} P_{\beta}\right)\right\}=\left\{g_{i j}, \int d^{3} x\left(N H+N^{m} H_{m}\right)\right\}, \\
p^{i j}{ }_{, t} & =\left\{p^{i j}, \int d^{3} x\left(N H+N^{m} H_{m}+\Lambda^{\beta} P_{\beta}\right)\right\}=\left\{p^{i j}, \int d^{3} x\left(N H+N^{m} H_{m}\right)\right\}, \\
H & =0, \\
H_{i} & =0,
\end{aligned}
$$

supplemented by the expected equations for the foliation variables:

$$
\begin{aligned}
Y_{, t}^{\alpha} & =\left\{Y^{\alpha}, \int d^{3} x\left(N H+N^{m} H_{m}+\Lambda^{\beta} P_{\beta}\right)\right\}=\Lambda^{\alpha}, \\
P_{\alpha, t} & =\left\{P_{\alpha}, \int d^{3} x\left(N H+N^{m} H_{m}+\Lambda^{\beta} P_{\beta}\right)\right\}=0, \\
P_{\alpha} & =0 .
\end{aligned}
$$

\section{Equivalence classes of solutions and the sets $\Gamma$ and $\Delta$}

This section investigates the set of solutions, the set of Diff $\mathcal{M}$-classes of solutions, and the set of first-class orbits of the extended action. The many-to-one relationship that exists between the last two sets forms the backbone of the covariant canonical formalism.

\subsection{The set of solutions}

Let us first summarise the content of the extended formalism. The spacetime action (30) depends on the metric $\mathbf{G}$, the mappings $\mathbf{T}$ and $\mathbf{X}$, the induced fields $N, N^{i}$ and $g$ on $\mathbb{R} \times \Sigma$ and the multipliers $\mathbf{M}$. The set Virt $\mathcal{M}$ of all kinematically admissible configurations of the theory includes the appropriate restrictions for the fields $N, N^{i}$ and $g$ on $\mathbb{R} \times \Sigma$ in order to ensure compatibility between $\mathbf{T}, \mathbf{X}$ and $\mathbf{G}$ at the level of the solutions. The field equations derived from (30) imply that the multipliers $\mathbf{M}$ vanish, the metric $\mathbf{G}$ satisfies the Einstein vacuum equations, and the remaining fields $\mathbf{T}, \mathbf{X}, N, N^{i}$ and $g$ are related to the solution $\mathbf{G}$ through equation (26). Therefore, each solution $s \in$ Sol $\mathcal{M}$ can be equivalently described by the set of fields

$$
s=(\mathbf{G}, \mathbf{T}, \mathbf{X})
$$


provided that $\mathbf{G}$ satisfies the Einstein vacuum equations, and $\mathbf{T}$ and $\mathbf{X}$ are compatible with $\mathbf{G}$ in the sense that their gradients are respectively timelike and spacelike. Within the framework of the canonical action (44), each solution $s \in \operatorname{Sol}(\mathbb{R} \times \Sigma)$ can be described by the set

$$
s=(g, p, Y),
$$

provided that $g$ and $p$ and $Y$ satisfy the field equations of (44). The connection between the spacetime and the canonical representations of solutions is the following: Given a solution $(g, p, Y) \in \operatorname{Sol}(\mathbb{R} \times \Sigma)$ of (44), the lapse $N$ and the shift $N^{i}$ can be in principle calculated from $g$ and $p$, determining a solution

$$
s=\left(g, N, N^{i}, Y\right)
$$

of the field equations of the Lagrangian action (43). Then, the solution $(\mathbf{G}, \mathbf{T}, \mathbf{X}) \in \operatorname{Sol} \mathcal{M}$ of the spacetime action (30), constructed from $\left(g, N, N^{i}, Y\right)$, is such that the mappings $\mathbf{T}$ and $\mathbf{X}$, inverse to $Y$, are compatible with $\mathbf{G}$.

\subsection{The set $\Gamma$}

Regardless of representation, an equivalence relationship exists between the solutions of any version of the extended action. In the context of the spacetime action (30), two solutions $\left(\mathbf{G}_{1}, \mathbf{T}_{1}, \mathbf{X}_{1}\right)$ and $\left(\mathbf{G}_{2}, \mathbf{T}_{2}, \mathbf{X}_{2}\right)$ are equivalent,

$$
\left(\mathbf{G}_{1}, \mathbf{T}_{1}, \mathbf{X}_{1}\right) \sim\left(\mathbf{G}_{2}, \mathbf{T}_{2}, \mathbf{X}_{2}\right),
$$

if there exists a diffeomorphism $D: \mathcal{M} \rightarrow \mathcal{M}$ such that

$$
\mathbf{G}_{2}=D_{*} \mathbf{G}_{1}, \mathbf{T}_{2}=D_{*} \mathbf{T}_{1}, \mathbf{X}_{2}=D_{*} \mathbf{X}_{1},
$$

where $D_{*}$ is the push-forward mapping. Each equivalence class of solutions determines a unique element $\gamma \in \Gamma$, which can be denoted by

$$
\gamma=\{(\mathbf{G}, \mathbf{T}, \mathbf{X})\} .
$$

In the context of the canonical action (44), the classification of the elements of $\Gamma$ looks actually simpler: Two solutions $\left(g_{1}, p_{1}, Y_{1}\right)$ and $\left(g_{2}, p_{2}, Y_{2}\right)$ of (44) are equivalent,

$$
\left(g_{1}, p_{1}, Y_{1}\right) \sim\left(g_{2}, p_{2}, Y_{2}\right),
$$

if they have the same components $g$ and $p$,

$$
g_{1}=g_{2}, p_{1}=p_{2},
$$

and differ only in their component $Y$. This fact follows from the covariance of the fields $\mathbf{G}, \mathbf{T}$ and $\mathbf{X}$ under spacetime diffeomorphisms, which leads to the invariance of the fields $g$ and $p$. Hence, each element $\gamma \in \Gamma$ can be denoted by

$$
\gamma=(g, p),
$$


or, equivalently, by

$$
\gamma=\left(g, N, N^{i}\right) .
$$

It should be noticed that the set $\Gamma$ of the extended action is isomorphic to the $\operatorname{set}^{\operatorname{Sol}^{\mathrm{ADM}}}(\mathbb{R} \times \Sigma)$ of solutions of the Dirac-ADM action.

\subsection{The set $\Delta$}

The set $\Delta$ of the extended action coincides with the corresponding set of the Dirac-ADM action due to the presence of the additional first-class constraints in (44). The equivalence relation that connects the sets $\Gamma$ and $\Delta$ of $(30)$ is, as expected, the following: Two elements $\gamma_{1}=\left\{\left(\mathbf{T}_{1}, \mathbf{X}_{1}, \mathbf{G}_{1}\right)\right\}$ and $\gamma_{2}=\left\{\left(\mathbf{T}_{2}, \mathbf{X}_{2}, \mathbf{G}_{2}\right)\right\}$ of the set $\Gamma$ are equivalent,

$$
\gamma_{1} \sim \gamma_{2}
$$

if the metric classes contained in $\gamma_{1}$ and $\gamma_{2}$ are the same,

$$
\left\{\mathbf{G}_{1}\right\}=\left\{\mathbf{G}_{2}\right\} .
$$

In other words, in order to obtain the set $\Delta$ from the set $\Gamma$ of the extended action one has to treat the mappings $\mathbf{T}$ and $\mathbf{X}$ as unimportant elements of the theory.

In the canonical representation, the same applies to the foliation mapping $Y$. Specifically, two elements $\gamma_{1}=\left(g_{1}, p_{1}\right)$ and $\gamma_{1}=\left(g_{1}, p_{1}\right)$ of the set $\Gamma$ are considered equivalent,

$$
\left(g_{1}, p_{1}\right)=\left(g_{2}, p_{2}\right),
$$

if the classes $\left\{\mathbf{G}_{1}\right\}$ and $\left\{\mathbf{G}_{1}\right\}$, constructed from $\left(g_{1}, p_{1}\right)$ and $\left(g_{2}, p_{2}\right)$ via arbitrary choices of the mapping $Y$, are the same. Thus, the relegation of the fields $\mathbf{T}, \mathbf{X}$ and $Y$ of the extended action to secondary elements of the theory is equivalent, in meaning, to the standard treatment of the foliation as an external element of the Dirac-ADM theory.

Summarising the findings of this section, the set $\Delta$ of the extended action is isomorphic to the set $\Delta^{\mathrm{ADM}}$ of the Dirac-ADM action and the set $\Gamma^{\mathrm{Hil}}$ of the Hilbert action. However, the set $\Gamma$ of the extended action is enlarged, because each element $\delta \in \Delta$ represents a whole class $\{\gamma\}$ of elements in $\Gamma$, making the correspondence between the sets $\Gamma$ and $\Delta$ many-to-one.

\section{$6 \quad$ Symmetries}

A generalised symmetry of the field equations is an infinitesimal transformation of the set Virt $\mathcal{M}$ that preserves its subset $\operatorname{Sol} \mathcal{M}$ of solutions. In general, the generator $\Delta \Psi[\Psi]$ of this transformation is a functional of all the field variables $\Psi$. This generator satisfies the linearised form of the field equations provided that these equations hold. Such symmetries map solutions in Sol $\mathcal{M}$ to other 'neighbouring' solutions. All local generalised symmetries of vacuum general 
relativity have been found by Anderson and Torre [64]. These consists of generalised spacetime diffeomorphisms and the constant scaling of the spacetime metric. Of course, the extension of the Hilbert action by the mappings $\mathbf{T}$ and $\mathbf{X}$ implies additional symmetries, the most important of which will be investigated in this section.

Overall, the symmetries that will be examined are split into two categories. The first category consists of symmetries induced by the diffeomorphisms of the manifolds $\mathcal{M}, \Sigma$ and $\mathbb{R}$. These are generated by vector fields $V^{\alpha}, v^{i}$ and $w$, that are regarded, respectively, as elements of the Lie algebras LDiff $\mathcal{M}, \mathrm{LDiff} \Sigma$ and LDiffR. Each of these groups of diffeomorphisms moves the elements of its own manifold and keeps the elements of the other manifolds fixed. Consequently, the transformations of the connecting mappings $\mathbf{T}, \mathbf{X}$ and $Y$ are especially important because they determine how the fields on $\mathcal{M}$ transform under Diff $\Sigma$ and Diff $\mathbb{R}$, and how the fields on $\Sigma \times \mathbb{R}$ transform under Diff $\mathcal{M}$.

The second category of symmetries consists of generalised changes $\delta \mathbf{T}[\boldsymbol{\Psi}]$ and $\delta \mathbf{X}[\boldsymbol{\Psi}], \boldsymbol{\Psi}=$ $(\mathbf{G}, \mathbf{T}, \mathbf{X})$, of the time and space mappings, where the events $y \in \mathcal{M}$ and the spacetime metric $\mathbf{G}$ are kept fixed, as well as generalised changes $\delta Y[g, p, Y]$ of the foliation mapping, where the moments $t \in \mathbb{R}$, the points $x \in \Sigma$ and the fields $g$ and $p$ are kept fixed. There are certain similarities between these two categories of symmetries which will be spelled out towards the end of this section. For simplicity, only the main variables will be considered; namely the spacetime fields $\mathbf{G}_{\alpha \beta}, \mathbf{T}$ and $\mathbf{X}$, the induced fields $g, N, N^{i}$, and $Y$, the momenta $p$ and $P$, and the canonical multipliers $\Lambda$. The remaining multipliers $M^{\alpha \beta}, M^{i j}, M^{i}$ and $M$ are not present in the covariant canonical action (44), so they will be left out of this discussion.

\subsection{Infinitesimal symmetries induced by $\operatorname{Diff} \mathcal{M}$}

These symmetries are generated by vector fields $V^{\alpha}$ on $\mathcal{M}$, and are defined by

$$
\begin{aligned}
\delta y^{\alpha} & :=V^{\alpha} \\
\delta x^{i} & :=0 \\
\delta t & :=0 .
\end{aligned}
$$

The spacetime fields respond to these changes via their Lie derivative $\mathcal{L}_{V}$ :

$$
\begin{aligned}
\delta \mathbf{G}_{\alpha \beta} & =-\mathcal{L}_{V} \mathbf{G}_{\alpha \beta}=-\mathbf{G}_{\alpha \beta, \gamma} V^{\gamma}-\mathbf{G}_{\alpha \gamma} V_{, \beta}^{\gamma}-\mathbf{G}_{\gamma \beta} V_{, \alpha}^{\gamma}, \\
\delta \mathbf{T} & =-\mathcal{L}_{V} \mathbf{T}=-\mathbf{T}_{, \gamma} V^{\gamma} \\
\delta \mathbf{X}^{i} & =-\mathcal{L}_{V} \mathbf{X}^{i}=-\mathbf{X}_{, \gamma}^{i} V^{\gamma} .
\end{aligned}
$$

The response of the connecting mapping $Y$ is determined by the condition $Y(\mathbf{T}, \mathbf{X})=\operatorname{Id}_{\mathcal{M}}$, where $\operatorname{Id}_{\mathcal{M}}$ is the identity transformation of $\mathcal{M}$. Infinitesimally, this relation becomes

$$
\delta Y^{\alpha}=-Y_{, t}^{\alpha} \delta \mathbf{T}(Y)-Y_{, i}^{\alpha} \delta \mathbf{X}^{i}(Y)
$$


Using (69)-(171) and the definitions (23)-(25) of the fields $g, N$ and $N^{i}$ in terms of the spacetime variables, the following changes are determined:

$$
\begin{aligned}
\delta Y^{\alpha} & =V^{\alpha}(Y), \\
\delta g_{i j} & =0, \\
\delta N & =0, \\
\delta N^{i} & =0 .
\end{aligned}
$$

In addition, the field equations derived from the canonical theory (44) and the relations (73)(75) imply that the momenta $p$ remain unchanged:

$$
\delta p^{i j}=0 .
$$

Finally, regarding the embedding momenta $P$ and the Lagrange multipliers $\Lambda$ in (44), one has to rely again on the field equations. The following changes then arise:

$$
\begin{aligned}
& \delta P_{\alpha}=0, \\
& \delta \Lambda^{\alpha}=\Lambda^{\beta} V_{, \beta}^{\alpha}(Y) .
\end{aligned}
$$

The geometric meaning of these changes, with the emphasis placed on the connecting mappings, was originally discussed in Ref. [1]: While spacetime diffeomorphisms do not move the moments $t$ in $\mathbb{R}$, they change the time map $\mathbf{T}$ and hence the foliation $\Sigma^{\mathbf{T}}$ in $\mathcal{M}$. They send the instant $\Sigma_{(t)}^{\mathbf{T}}$ of the original time foliation $\Sigma^{\mathbf{T}}$ onto the instant $\Sigma_{(t)}^{\mathbf{T}+\delta \mathbf{T}}$ of a different time

foliation $\Sigma^{\mathbf{T}+\delta \mathbf{T}}$ : In general, the instant $\Sigma_{(t)}^{\mathbf{T}+\delta \mathbf{T}}$ is not only different from the instant $\Sigma_{(t)}^{\mathbf{T}}$, but also from all the other instants $\Sigma_{\left(t^{\prime}\right)}^{\mathbf{T}}, t^{\prime} \in \mathbb{R}$, of the original time foliation $\Sigma^{\mathbf{T}}$. Similarly, while spacetime diffeomorphisms do not move the points $x$ in $\Sigma$, they change the map $\mathbf{X}$ and hence the reference frame $C^{\mathbf{x}}$ in $\mathcal{M}$. They send the reference worldline $C_{(x)}^{X}$ of the original reference frame $C^{\mathbf{X}}$ onto the reference worldline $C_{(x)}^{\mathbf{X}+\delta \mathbf{X}}$ of a different reference frame $C^{\mathbf{X}+\delta \mathbf{X}}$ : In general, the reference worldline $C_{(x)}^{\mathbf{X}+\delta \mathbf{X}}$ is not only different from the reference worldline $C_{(x)}^{\mathbf{X}}$, but also from all the other reference worldlines $C_{\left(x^{\prime}\right)}^{\mathbf{X}}, x^{\prime} \in \Sigma$, of the original reference frame $C^{\mathbf{x}}$.

The correlations $g$ and $p$ between the original metric $\mathbf{G}$ and the original time foliation $\Sigma^{\mathbf{T}}$ and reference frame $C^{\mathbf{X}}$ are preserved, in the sense that the transformed metric $\mathbf{G}+\delta \mathbf{G}$ and the transformed time foliation $\Sigma^{\mathbf{T}+\delta \mathbf{T}}$ and reference frame $C^{\mathbf{X}+\delta \mathbf{X}}$ yield exactly the same correlations $g$ and $p$. Crucially, the compatibility conditions encoded in the set of virtual fields of the theory are respected. Indeed, since the fields $g, N$ and $N^{i}$ are left invariant by spacetime diffeomorphisms, (73)-(75) , a positive definite $g$ remains positive definite, or a positive $N$ remains positive.

\subsection{Infinitesimal symmetries induced by Diff $\Sigma$}

These symmetries are generated by vector fields $u^{i}$ on $\Sigma$, and are defined by

$$
\delta y^{\alpha}:=0,
$$




$$
\begin{aligned}
\delta x^{i} & :=u^{i} \\
\delta t & :=0
\end{aligned}
$$

The fields on $\mathbb{R} \times \Sigma$ respond to these changes via their Lie derivative $\mathcal{L}_{u}$ on $\Sigma$,

$$
\begin{aligned}
\delta Y^{\alpha} & =-\mathcal{L}_{u} Y^{\alpha}=-Y_{, i}^{\alpha} u^{i}, \\
\delta g_{i j} & =-\mathcal{L}_{u} g_{i j}=-g_{i j, k} u^{k}-g_{i k} u_{, j}^{k}-g_{k j} u_{, i}^{k}, \\
\delta N & =-\mathcal{L}_{u} N=-N_{, i} u^{i}, \\
\delta N^{i} & =-\mathcal{L}_{u} N^{i}=-N_{, j}^{i} u^{j}+N^{j} u_{, j}^{i}, \\
\delta p^{i j} & =-\mathcal{L}_{u} p^{i j}=-p_{, k}^{i j} u^{k}+p^{i k} u_{, k}^{j}+p^{k j} u_{, k}^{i}-p^{i j} u_{, k}^{k}, \\
\delta P_{\alpha} & =0, \\
\delta \Lambda^{\alpha} & =-\mathcal{L}_{u} \Lambda^{\alpha}=-\Lambda_{, k}^{\alpha} u^{k},
\end{aligned}
$$

where it should be recalled that the momenta $p^{i j}$ are densities of weight one on $\Sigma$. The responses of the connecting mappings $\mathbf{T}$ and $\mathbf{X}$ are determined by the conditions $\mathbf{T}(Y)=\operatorname{Id}_{\mathbb{R}}$ and $\mathbf{X}(Y)=\operatorname{Id}_{\Sigma}$, where $\operatorname{Id}_{\mathbb{R}}$ and $\operatorname{Id}_{\Sigma}$ are the identity transformations of $\mathbb{R}$ and $\Sigma$. Infinitesimally, these relations imply that

$$
\begin{aligned}
\delta \mathbf{T} & =-\mathbf{T}_{, \alpha} \delta Y^{\alpha}(\mathbf{T}, \mathbf{X}) \\
\delta \mathbf{X}^{i} & =-\mathbf{X}_{, \alpha}^{i} \delta Y^{\alpha}(\mathbf{T}, \mathbf{X})
\end{aligned}
$$

which, when combined with (82), yield

$$
\begin{aligned}
\delta \mathbf{T} & =0 \\
\delta \mathbf{X}^{i} & =u^{i}(\mathbf{X})
\end{aligned}
$$

Using the changes (83)-(85) and (91)-(92) together with the definition (26)-(29) of $\mathbf{G}$ in terms of $g, N, N^{i}, \mathbf{T}$ and $\mathbf{X}$, it follows that $\mathbf{G}$ remains invariant:

$$
\delta \mathbf{G}_{\alpha \beta}=0
$$

As discussed in Ref. [1], space diffeomorphisms send the reference worldline $C_{(x)}^{\mathbf{X}}$ of the original reference frame $C^{\mathbf{X}}$ to the reference worldline $C_{(x+\delta x)}^{\mathbf{X}+\delta \mathbf{X}}$ of the same reference frame $C^{\mathbf{X}}$; i.e., the final reference frame is exactly the same collection of reference wordlines in $\mathcal{M}$ as the original. In addition, space diffeomorphisms do not change the time map $\mathbf{T}$, so they leave, not only the time foliation $\Sigma^{\mathbf{T}}$ but also its individual instants $\Sigma_{(t)}^{\mathbf{T}}$, fixed. While the spacetime metric field $\mathbf{G}$ is left unchanged, the induced fields on $\mathbb{R} \times \Sigma$ are transformed due to the relabeling of the wordlines of each reference frame. Regarding the compatibility conditions, these are respected on account of the fact that each reference frame and each time foliation is left unchanged, as $\mathrm{G}$ is. 


\subsection{Infinitesimal symmetries induced by Diff $\mathbb{R}$}

These symmetries are generated by vector fields $w$ on $\mathbb{R}$, and are defined by

$$
\begin{aligned}
\delta y^{\alpha} & :=0 \\
\delta x^{i} & :=0 \\
\delta t & :=w .
\end{aligned}
$$

The fields on $\mathbb{R} \times \Sigma$ respond to these changes via their Lie derivative $\mathcal{L}_{w}$ on $\mathbb{R}$,

$$
\begin{aligned}
\delta Y^{\alpha} & =-\mathcal{L}_{w} Y^{\alpha}=-Y_{, t}^{\alpha} w \\
\delta g_{i j} & =-\mathcal{L}_{w} g_{i j}=-g_{i j, t} w \\
\delta N & =-\mathcal{L}_{w} N=-N_{, t} w-N w_{, t} \\
\delta N^{i} & =-\mathcal{L}_{w} N^{i}=-N_{, t}^{i} w-N^{i} w_{, t} \\
\delta p^{i j} & =-\mathcal{L}_{w} p^{i j}=-p_{, t}^{i j} w \\
\delta P_{\alpha} & =0 \\
\delta \Lambda^{\alpha} & =-\mathcal{L}_{w} \Lambda^{\alpha}=-\Lambda_{, t}^{\alpha} w-\Lambda^{\alpha} w_{, t}
\end{aligned}
$$

where it should be recalled that the multipliers $N, N^{i}$ and $\Lambda$ are densities of weight one on $\mathbb{R}$. The responses of the connecting mappings $\mathbf{T}$ and $\mathbf{X}$ are determined using the relationships (89)-(90) which, when combined with (97), yield

$$
\begin{aligned}
\delta \mathbf{T} & =w(\mathbf{T}), \\
\delta \mathbf{X}^{i} & =0 .
\end{aligned}
$$

Using (98)-(100) and (102)-(105) together with the definition (26)-(29) of $\mathbf{G}$ in terms of $g, N$, $N^{i}, \mathbf{T}$ and $\mathbf{X}$, it follows again that $\mathbf{G}$ remains invariant:

$$
\delta \mathbf{G}_{\alpha \beta}=0
$$

Regarding the interpretation of these changes [1], time diffeomorphisms send the instant $\Sigma_{(t)}^{\mathbf{T}}$ of the original time foliation $\Sigma^{\mathbf{T}}$ onto the instant $\Sigma_{(t+\delta t)}^{\mathbf{T}+\delta \mathbf{T}}$ of the same time foliation $\Sigma^{\mathbf{T}}$. Although time diffeomorphisms relabel the instants of a given time foliation, the final time foliation consists of exactly the same collection of instants in $\mathcal{M}$ as the original. In addition, time diffeomorphisms do not affect the space map $\mathbf{X}$, and hence they leave, not only the reference frame $C^{\mathbf{X}}$ but also its individual reference worldlines $C_{(x)}^{\mathbf{X}}$, fixed. While the spacetime metric field $\mathbf{G}$ is left unchanged, the induced fields on $\mathbb{R} \times \Sigma$ are transformed due to the relabeling of the instants of each time foliation. The compatibility conditions are again respected on account of the fact that each reference frame and each time foliation is left unchanged, as $\mathbf{G}$ is. 


\subsection{Infinitesimal symmetries induced by deformations of $\mathrm{T}$ and $\mathrm{X}$}

The action of space diffeomorphisms and time diffeomorphisms on the manifold points,

$$
\delta y=0, \delta t=w(t), \delta x^{i}=u^{i}(x),
$$

and the connecting mappings,

$$
\delta Y=-Y_{, t} w-Y_{, i} u^{i}, \delta \mathbf{T}=w(\mathbf{T}), \delta \mathbf{X}^{i}=u^{i}(\mathbf{X}),
$$

can be considered as a single action, which is equivalent to a transformation of the mappings $\mathbf{T}$ and $\mathbf{X}$ while the events $y \in \mathcal{M}$ and the spacetime metric $\mathbf{G}$ are kept fixed. As we have seen, such a transformation is quite special because it only relabels the hypersurfaces of the time foliation, and the wordlines of the reference frame, without deforming either of them. More general transformations of the mappings $\mathbf{T}$ and $\mathbf{X}$ can now be examined, which generally deform both the time foliation and the reference frame. However, even if such changes formally preserve the field equations derived from the extended action, they may actually fail to be symmetries of the formalism. This is because they may violate the restrictions imposed on the virtual fields $\left(g, N, N^{i}\right)$ of the theory, necessary in order to ensure compatibility between $\mathbf{T}, \mathbf{X}$ and $\mathbf{G}$ at the level of the solutions.

For example, if changes of the form $\delta \mathbf{T}[\mathbf{T}, \mathbf{X}]$ and $\delta \mathbf{X}[\mathbf{T}, \mathbf{X}]$ are considered, which depend on the mappings $\mathbf{T}, \mathbf{X}$ but not on $\mathbf{G}$, then these changes will certainly violate, at least for some subset of solutions $(\mathbf{G}, \mathbf{T}, \mathbf{X})$, the compatibility conditions between $\mathbf{T}, \mathbf{X}$ and $\mathbf{G}$. Indeed, given changes $\delta \mathbf{T}[\mathbf{T}, \mathbf{X}], \delta \mathbf{X}[\mathbf{T}, \mathbf{X}]$ that deform the time foliation and reference frame while leaving the spacetime metric unchanged, one can always find a solution $\mathbf{G}$ that is compatible with the original pair $(\mathbf{T}, \mathbf{X})$ but not with the final pair $(\mathbf{T}+\delta \mathbf{T}, \mathbf{X}+\delta \mathbf{X})$. In this case, the corresponding final variables $\left(g+\delta g, N+\delta N, N^{i}+\delta N^{i}\right)$ will no longer belong to the set of virtual fields of the theory; i.e, a positive definite $g$ will no longer be positive definite, etc.

On the other hand, if the deformations are allowed to depend on all the fields $\mathbf{T}, \mathbf{X}$ and $\mathbf{G}$, then it is indeed possible, at least in principle, to define symmetries $\delta \mathbf{T}[\mathbf{T}, \mathbf{X}, \mathbf{G}]$ and $\delta \mathbf{X}[\mathbf{T}, \mathbf{X}, \mathbf{G}]$ of the field equations of the extended action. The corresponding infinitesimal changes induced on the field variables are evaluated below. Their finite counterparts are not expected to form a group; see Pitts and Schieve for a similar phenomenon [65]. For notational simplicity, the square bracket indicating the functional dependence of the infinitesimal changes $\delta \mathbf{T}$ and $\delta \mathbf{X}$ on the field variables is not used, but it should be kept in mind that $\delta \mathbf{T}$ and $\delta \mathbf{X}$ are now functions on both $\mathbb{R}$ and $\Sigma$; i.e., they depend on both $t$ and $x$.

By definition, under these generalised changes $\delta \mathbf{T}, \delta \mathbf{X}$, we have that

$$
\begin{aligned}
\delta y^{\alpha} & :=0, \\
\delta \mathbf{G} & :=0, \\
\delta x^{i} & :=\delta \mathbf{X}^{i}(t, x), \\
\delta t & :=\delta \mathbf{T}(t, x) .
\end{aligned}
$$


The fields on $\mathbb{R} \times \Sigma$ respond to these changes via their Lie derivatives $\mathcal{L}_{\delta \mathbf{T}}$ on $\mathbb{R}$ and $\mathcal{L}_{\delta \mathbf{X}}$ on $\Sigma$ together with some additional terms of both kinematical and dynamical origin:

$$
\begin{aligned}
\delta Y^{\alpha} & =-\mathcal{L}_{\delta \mathbf{T}} Y^{\alpha}-\mathcal{L}_{\delta \mathbf{X}} Y^{\alpha} \\
\delta g_{i j} & =-\mathcal{L}_{\delta \mathbf{T}} g_{i j}-\mathcal{L}_{\delta \mathbf{X}} g_{i j}-N_{i} \delta \mathbf{T}_{, j}-N_{j} \delta \mathbf{T}_{, i}, \\
\delta N & =-\mathcal{L}_{\delta \mathbf{T}} N-\mathcal{L}_{\delta \mathbf{X}} N+N N^{k} \delta \mathbf{T}_{, k}, \\
\delta N^{i} & =-\mathcal{L}_{\delta \mathbf{T}} N^{i}-\mathcal{L}_{\delta \mathbf{X}} N^{i}-\delta \mathbf{X}_{, t}^{i}+\left(N^{2} g^{i k}+N^{i} N^{k}\right) \delta \mathbf{T}_{, k}, \\
\delta \Lambda^{\alpha} & =-\mathcal{L}_{\delta \mathbf{T}} \Lambda^{\alpha}-\mathcal{L}_{\delta \mathbf{X}} \Lambda^{\alpha}-Y_{, i}^{\alpha} \delta \mathbf{X}_{, t}^{i} .
\end{aligned}
$$

These expressions reproduce the symmetries under Diff $\mathbb{R}$ in the special case where $\delta \mathbf{T}=$ $w(\mathbf{T})$ and $\delta \mathbf{X}=0$, and the symmetries under Diff $\Sigma$ in the special case where $\delta \mathbf{T}=0$ and $\delta \mathbf{X}=u(\mathbf{X})$. Next, using the field equations of the covariant canonical action (44), one can determine the changes of the canonical momenta. After some lengthy but straightforward calculation for the metric momenta, one finds that

$$
\begin{aligned}
\delta p^{i j}= & -\mathcal{L}_{\delta \mathbf{T}} p^{i j}-\mathcal{L}_{\delta \mathbf{x}} p^{i j}+\left(N^{i} p^{j k}+N^{j} p^{i k}-N^{k} p^{i j}\right) \delta \mathbf{T}_{, k} \\
& +g^{\frac{1}{2}}\left(2 g^{i j} g^{k l}-g^{i k} g^{j l}-g^{i l} g^{j k}\right) N_{, k} \delta \mathbf{T}_{, l}, \\
\delta P_{\alpha}= & 0 .
\end{aligned}
$$

The interpretation of these Bergmann-Komar [66] deformations is the following: The time foliation $\Sigma^{\mathbf{T}}$ and the reference frame $C^{\mathbf{X}}$ are mapped to an entirely different time foliation

$\Sigma^{\mathbf{T}+\delta \mathbf{T}}$ and an entirely different reference frame $C^{\mathbf{X}+\delta \mathbf{X}}$, while the solution $\mathbf{G}$ is kept fixed. The compatibility between the unchanged $\mathbf{G}$ and the transformed mappings $\mathbf{T}+\delta \mathbf{T}$ and $\mathbf{X}+\delta \mathbf{X}$ is preserved only if the functionals $\delta \mathbf{T}$ and $\delta \mathbf{X}$ generate changes (114)-(116) that preserve the restrictions imposed on the set of virtual fields of the theory. Provided that this is the case, the transformed metric $g+\delta g$ is positive definite, and so on.

\subsection{Infinitesimal symmetries induced by deformations of $Y$}

A symmetrical treatment of the spacetime and the canonical versions of the extended action suggests that one should also consider generalised deformations $\delta Y[g, p, Y]$ of the foliation mapping, where the moments $t \in \mathbb{R}$, the points $x \in \Sigma$ and the fields $g$ and $p$ on $\mathbb{R} \times \Sigma$ are kept fixed. However, these Bergmann-Komar changes are equivalent to generalised spacetime diffeomorphisms, where a different diffeomorphism is performed in each solution spacetime. Therefore, the infinitesimal changes induced on the field variables of the extended action are identical in form to the ones that have been already considered in the relevant part of this section. The only modification that needs to be made in order to incorporate these symmetries in the extended framework is to replace the Diff $\mathcal{M}$-induced changes $\delta Y=V(Y)$ by the generalised vector field $\delta Y[g, p, Y]$. 


\section{The action of symmetries on the phase space}

In this section, the extended equal-time phase space $\mathcal{P}=\{g, p, Y, P\}$ is introduced, and the action of symmetries on $\mathcal{P}$ is investigated.

\subsection{Symplectic form and solutions as curves in $\mathbb{R} \times \mathcal{C}$}

The standard symplectic form on the phase space $\mathcal{P}$ of the covariant-canonical action (44) yields the fundamental Poisson brackets

$$
\begin{aligned}
\left\{g_{i j}(x), p^{m n}\left(x^{\prime}\right)\right\} & =\delta_{i j}^{m n} \delta\left(x, x^{\prime}\right) \\
\left\{Y^{\alpha}(x), P_{\beta}\left(x^{\prime}\right)\right\} & =\delta_{\beta}^{\alpha} \delta\left(x, x^{\prime}\right)
\end{aligned}
$$

where $\delta_{i j}^{m n}:=\frac{1}{2}\left(\delta_{i}^{m} \delta_{j}^{n}+\delta_{j}^{m} \delta_{i}^{n}\right)$. The Dirac delta function $\delta\left(x, x^{\prime}\right)$ is a scalar function on $\Sigma$ in the first argument and a scalar density on $\Sigma$ in the second argument. All the remaining Poisson brackets between the fields $g, p, Y$ and $P$ are zero.

Although this is not essential, it is nonetheless clearer to consider the product space $\mathbb{R} \times \mathcal{P}$, which is the natural space where solutions lie. Specifically, each solution $(g(t), p(t), Y(t), P(t))$ of (44) is viewed as a one-parameter family $\left(g_{(t)}, p_{(t)}, Y_{(t)}, P_{(t)}\right)$ of instantaneous data; that is, as a curve in $\mathbb{R} \times \mathcal{P}$. Permissible instantaneous data lie on the constraint surface $\mathcal{C} \subset \mathcal{P}$ of (44) determined by the super-Hamiltonian and super-momentum constraints (47)-(48), and the annihilation of the embedding momenta, (51). The curves representing the solutions of (44) lie on the subspace $\mathbb{R} \times \mathcal{C}$ of $\mathbb{R} \times \mathcal{P}$, and each moment $t_{o} \in \mathbb{R}$ defines a copy $t_{o} \times \mathcal{C} \in \mathbb{R} \times \mathcal{C}$ of the constraint surface $\mathcal{C}$.

The primary role of the Hamiltonian

$$
\int d^{3} x \mathcal{H}:=\int d^{3} x\left(N H+N^{i} H_{i}+\Lambda^{\alpha} P_{\alpha}\right)
$$

of (44) is to generate the dynamical evolution of the system. More precisely, $\int d^{3} x \mathcal{H}$ generates a curve $\left(g_{(t)}, p_{(t)}, Y_{(t)}, P_{(t)}\right)$ in $\mathbb{R} \times \mathcal{C}$ from an initial datum $\left(g_{\left(t_{o}\right)}, p_{\left(t_{o}\right)}, Y_{\left(t_{o}\right)}, P_{\left(t_{o}\right)}\right)$ in $t_{o} \times \mathcal{C}$ via its Poisson bracket action

$$
f_{\left(t_{o}+\delta t\right)}(x)=f_{\left(t_{o}\right)}(x)+\delta f_{\left(t_{o}\right)}(x)=f_{\left(t_{o}\right)}(x)+\left.\left\{f(x), \int d^{3} x \mathcal{H}\right\}\right|_{f=f_{\left(t_{o}\right)}} \delta t,
$$

where $f$ denotes any of the canonical variables. The symbol $\left.\{\}\right|_{,f=f_{\left(t_{o}\right)}}$ means that the phasespace fields $(g, p, Y, P)$ must be evaluated at the particular initial datum $\left(g_{\left(t_{o}\right)}, p_{\left(t_{o}\right)}, Y_{\left(t_{o}\right)}, P_{\left(t_{o}\right)}\right)$ after the Poisson brackets have been worked out. The multipliers $N, N^{i}$ and $\Lambda$ appearing in (123) are regarded as smearing functions on $\Sigma$; i.e, they carry no $t$-dependence at this stage. Different choices of such functions generate different curves in $\mathbb{R} \times \mathcal{C}$. 


\subsection{The action of symmetries on solutions}

Assuming that all solutions, i.e., complete dynamical trajectories of (44), have been generated by $\mathcal{H}$ via (123), the multipliers $N, N^{i}$ and $\Lambda$ now become functions of $t$ : that is, for each given solution $\left(g_{(t)}, p_{(t)}, Y_{(t)}, P_{(t)}\right)$ in $\mathbb{R} \times \mathcal{C}$, the multipliers can be in principle evaluated as functions of $t$ using the field equations. A symmetry of the field equations of (44) acts on each one of these solutions according to the expressions derived in section 5. It maps each curve

$$
\left(g_{(t)}, p_{(t)}, Y_{(t)}, P_{(t)}\right) \in \mathbb{R} \times \mathcal{C}
$$

to a neighbouring curve

$$
\left((g+\delta g)_{(t)},(p+\delta p)_{(t)},(Y+\delta Y)_{(t)},(P+\delta P)_{(t)}\right) \in \mathbb{R} \times \mathcal{C},
$$

where the changes $\delta g_{(t)}, \delta p_{(t)}, \delta Y_{(t)}$ and $\delta P_{(t)}$ depend in general on the particular curve and symmetry considered. The actions of the diffeomorphisms of $\mathcal{M}, \Sigma$ and $\mathbb{R}$ and the action of the generalised deformations $\delta \mathbf{T}, \delta \mathbf{X}$ and $\delta Y$ on these curves have been already investigated in section 5. In this section, the dynamical variables in $\mathcal{P}$ that generate these actions are identified.

\subsection{The canonical generator of $\operatorname{Diff} \mathcal{M}$}

For convenience, let us summarise the changes induced by Diff $\mathcal{M}$ on each solution curve in $\mathbb{R} \times \mathcal{C}$. Copying these changes from section 5 , we have:

$$
\begin{aligned}
\delta g_{i j} & =0, \\
\delta p^{i j} & =0, \\
\delta Y^{\alpha} & =V^{\alpha}(Y), \\
\delta P_{\alpha} & =-P_{\beta} V_{, \alpha}^{\beta}(Y) .
\end{aligned}
$$

If $f_{(t)}(x)$ denotes any of these solution components, the general change $\delta f_{(t)}(x)$ in $\mathbb{R} \times \mathcal{C}$ is weakly reproduced via the Poisson brackets

$$
\delta f_{(t)}(x)=\left.\left\{f(x), \mathcal{D}_{V}\right\}\right|_{f(x)=f_{(t)}(x)}
$$

of $f(x)$ with the dynamical variable

$$
\mathcal{D}_{V}=\int d^{3} x P_{\alpha}(x) V^{\alpha}(Y(x))
$$

The generator $\mathcal{D}_{V}$ depends solely on the embedding variables and the generating vector field $V$, and therefore has no counterpart in the Dirac-ADM theory. Moreover, the ultra-locality of $\mathcal{D}_{V}$ in $t$; i.e., the fact that $\mathcal{D}_{V}$ does not contain any $t$-derivatives, implies that the action of this generator on $\mathbb{R} \times \mathcal{C}$ depends only on the points $c \in \mathcal{C}$. Therefore, this action induces 
a well-defined action on $\mathcal{C}$, and spacetime diffeomorphisms can be represented by symplectic diffeomorphisms of the phase space. Specifically, the generator $\mathcal{D}_{V}$ defines an anti-homomorphic mapping from vector fields in the Lie algebra of Diff $\mathcal{M}$ into the Poisson bracket algebra on the phase space of the system:

$$
\left\{\mathcal{D}_{V_{1}}, \mathcal{D}_{V_{2}}\right\}=\mathcal{D}_{-\left[V_{1}, V_{2}\right]},
$$

where the Lie bracket $\left[V_{1}, V_{2}\right]$ of the vector fields $V_{1}$ and $V_{2}$ is defined by

$$
\left[V_{1}, V_{2}\right]^{\alpha}:=V_{1}^{\beta} V_{2, \beta}^{\alpha}-V_{2}^{\beta} V_{1, \beta}^{\alpha}
$$

The reason that this representation is established via an anti-homomorphism, rather than a homomorphism, has been discussed extensively in Refs. [12] and [1].

\subsection{The canonical generator of $\operatorname{Diff} \Sigma$}

The changes induced by Diff $\Sigma$ on each solution curve in $\mathbb{R} \times \mathcal{C}$ are:

$$
\begin{aligned}
\delta g_{i j} & =-g_{i j, k} u^{k}-g_{i k} u_{, j}^{k}-g_{k j} u_{, i}^{k}, \\
\delta p^{i j} & =-p_{, k}^{i j} u^{k}+p^{i k} u_{, k}^{j}+p^{k j} u_{, k}^{i}-p^{i j} u_{, k}^{k}, \\
\delta Y^{\alpha} & =-Y_{, i}^{\alpha} u^{i} \\
\delta P_{\alpha} & =-P_{\alpha, k} u^{k}-P_{\alpha} u_{, k}^{k} .
\end{aligned}
$$

Letting again $f_{(t)}(x)$ denote any of these solution components, the general change $\delta f_{(t)}(x)$ in $\mathbb{R} \times \mathcal{C}$ is weakly reproduced via the Poisson brackets

$$
\delta f_{(t)}(x)=\left.\left\{f(x), \mathcal{D}_{u}\right\}\right|_{f(x)=f_{(t)}(x)}
$$

of $f(x)$ with the dynamical variable

$$
\mathcal{D}_{u}=\int d^{3} x u^{i}(x)\left(H_{i}(x)+P_{\alpha}(x) Y_{, i}^{\alpha}(x)\right) .
$$

The generator $\mathcal{D}_{u}$ is ultralocal in $t$, which again provides the key to representing space diffeomorphism by symplectic diffeomorphisms of $\mathcal{P}$. Specifically, a homomorphism arises,

$$
\left\{\mathcal{D}_{u_{1}}, \mathcal{D}_{u_{2}}\right\}=\mathcal{D}_{\left[u_{1}, u_{2}\right]}
$$

where the Lie bracket $\left[u_{1}, u_{2}\right]$ of the vector fields $u_{1}$ and $u_{2}$ on $\Sigma$ is given by

$$
\left[u_{1}, u_{2}\right]^{i}:=u_{1}^{j} u_{2, j}^{i}-u_{2}^{j} u_{1, j}^{i}
$$




\subsection{The canonical generator of DiffR}

The changes induced by Diff $\mathbb{R}$ on each solution curve in $\mathbb{R} \times \mathcal{C}$ are:

$$
\begin{aligned}
\delta g_{i j} & =-g_{i j, t} w, \\
\delta p^{i j} & =-p_{, t}^{i j} w, \\
\delta Y^{\alpha} & =-Y_{, t}^{\alpha} w, \\
\delta P_{\alpha} & =-P_{\alpha, t} w .
\end{aligned}
$$

The general change $\delta f_{(t)}(x)$ in $\mathbb{R} \times \mathcal{C}$ induced by time diffeomorphisms is again weakly reproduced via the Poisson brackets

$$
\delta f_{(t)}(x)=\left.\left\{f(x), \mathcal{D}_{w}\right\}\right|_{f(x)=f_{(t)}(x)}
$$

of $f(x)$ with the dynamical variable

$$
\mathcal{D}_{w}=-w(t) \int d^{3} x \mathcal{H}(x),
$$

where $\int d^{3} x \mathcal{H}$ is the Hamiltonian (122). However, the smearing functions $N(x), N^{i}(x)$ and $\Lambda(x)$ that are present in (135) must be replaced at the end of the calculation by the lapse $N_{(t)}(x)$, shift $N_{(t)}^{i}(x)$ and multipliers $\Lambda_{(t)}(x)$ associated with the particular solution curve in $\mathbb{R} \times \mathcal{C}$ whose change is considered. This means that the action of the generator $\mathcal{D}_{w}$ on $\mathbb{R} \times \mathcal{C}$ depends not only on the point $c \in \mathcal{C}$, but also on the particular curve passing through this point. Hence, it does not induce a well-defined action on $\mathcal{C}$. In this case, the Lie algebra of vector fields on $\mathbb{R}$ cannot be represented by symplectic diffeomorphisms of $\mathcal{P}$. This is possible only within a history phase space formulation of the canonical action (44).

\subsection{The canonical generator of generalised deformations $\delta \mathbf{T}, \delta \mathbf{X}$}

The changes induced on each solution curve in $\mathbb{R} \times \mathcal{C}$ by the deformations $\delta \mathbf{T}$ and $\delta \mathbf{X}$ of the mappings are:

$$
\begin{aligned}
\delta g_{i j}= & -\mathcal{L}_{\delta \mathbf{T}} g_{i j}-\mathcal{L}_{\delta \mathbf{X}} g_{i j}-N_{i} \delta \mathbf{T}_{, j}-N_{j} \delta \mathbf{T}_{, i}, \\
\delta p^{i j}= & -\mathcal{L}_{\delta \mathbf{T}} p^{i j}-\mathcal{L}_{\delta \mathbf{X}} p^{i j}+\left(N^{i} p^{j k}+N^{j} p^{i k}-N^{k} p^{i j}\right) \delta \mathbf{T}_{, k} \\
& +g^{\frac{1}{2}}\left(2 g^{i j} g^{k l}-g^{i k} g^{j l}-g^{i l} g^{j k}\right) N_{, k} \delta \mathbf{T}_{, l}, \\
\delta Y^{\alpha}= & -\mathcal{L}_{\delta \mathbf{T}} Y^{\alpha}-\mathcal{L}_{\delta \mathbf{X}} Y^{\alpha}, \\
\delta P_{\alpha}= & 0 .
\end{aligned}
$$

The general change $\delta f_{(t)}(x)$ in $\mathbb{R} \times \mathcal{C}$ induced by time diffeomorphisms is weakly reproduced via the Poisson brackets

$$
\delta f_{(t)}(x)=\left.\left\{f(x), \mathcal{D}_{(\delta \mathbf{T}, \delta \mathbf{X})}\right\}\right|_{f(x)=f_{(t)}(x)}
$$


of $f(x)$ with the dynamical variable

$$
\mathcal{D}_{(\delta \mathbf{T}, \delta \mathbf{X})}=-\int d^{3} x\left(\delta \mathbf{T}\left(N H+N^{i} H_{i}+\Lambda^{\alpha} P_{\alpha}\right)+\delta \mathbf{X}^{i}\left(H_{i}+P_{\alpha} Y_{, i}^{\alpha}\right)\right) .
$$

Again, the smearing functions $N(x), N^{i}(x)$ and $\Lambda(x)$ that are present in (137) must be replaced at the end of the calculation by the lapse $N_{(t)}(x)$, shift $N_{(t)}^{i}(x)$ and multipliers $\Lambda_{(t)}(x)$ associated with the particular solution curve in $\mathbb{R} \times \mathcal{C}$ whose change is considered. Hence, it does not induce a well-defined action on $\mathcal{C}$. The representation of such deformations is again possible only within a history phase space formulation, an issue discussed in detail in Ref. 3]. Finally, it should be noted that the generator $\mathcal{D}_{(\delta \mathbf{T}, \delta \mathbf{X})}$ reduces to the generator $\mathcal{D}_{w}$ of time diffeomorphisms if $\delta \mathbf{T}=w(\mathbf{T})$ and $\delta \mathbf{X}=0$, and to the generator $\mathcal{D}_{u}$ of space diffeomorphisms if $\delta \mathbf{T}=0$ and $\delta \mathbf{X}=u(\mathbf{X})$.

\subsection{The canonical generator of generalised deformations $\delta Y$}

As discussed in the previous section, such deformations correspond to generalised spacetime diffeomorphisms. The general change $\delta f_{(t)}(x)$ in $\mathbb{R} \times \mathcal{C}$ of any solution component $f_{(t)}(x)$ is weakly reproduced via the Poisson brackets

$$
\delta f_{(t)}(x)=\left.\left\{f(x), \mathcal{D}_{\delta Y}\right\}\right|_{f(x)=f_{(t)}(x)}
$$

of $f(x)$ with the dynamical variable

$$
\mathcal{D}_{\delta Y}=\int d^{3} x P_{\alpha}(x) \delta Y^{\alpha}(x) .
$$

Of course, since $\delta Y^{\alpha}$ now depends upon all the canonical variables, it does not weakly commute with $g$ and $p$. Therefore, in order for (138)-(139) to reproduce the correct action on $g$ and $p$, explicit use of the constraint $P_{\alpha}=0$ must be made.

\section{Observables and their evolution}

An inspection of the canonical transformations of the previous section reveals two facts about canonical general relativity that are perhaps unexpected. First, although it is true that the invariance of the spacetime action under Diff $\mathcal{M}$ implies four first-class constraints, these are not the super-Hamiltonian and the super-momentum constraints but rather the embedding momentum constraints. Second, the orbits of the generator of spacetime diffeomorphism $\mathcal{D}_{\mathcal{M}}$ in the phase space are distinct from the orbits of the Hamiltonian $\int d^{3} x \mathcal{H}$. This eliminates any possibility of regarding the Hamiltonian as the generator of spacetime diffeomorphisms, in agreement with the long-standing viewpoint of Kuchar [39].

These results are analogous to the results obtained by Savvidou in the context of the history formulation of general relativity [2]-[4] and those obtained in Ref. [1] for the Bosonic string. 
In particular, the distinction between the gravitational Hamiltonian $\int d^{3} x \mathcal{H}$ and the generator of diffeomorphisms $\mathcal{D}_{\mathcal{M}}$ reflects analogous distinctions in [1]-4]. This has consequences for the observables of the theory and their dynamical evolution, a fact that, within the history framework, was pointed out in [3]-[4].

\subsection{Spacetime and Dirac observables}

In the present context, functions on the sets $\Gamma$ and $\Delta$ of (44) are connected with two kinds of instantaneous observables: spacetime observables and Dirac observables. In general, two functions $F_{1}$ and $F_{2}$ are defined to be equivalent on the phase space $\mathcal{P}$ of (44) if their values are equal on the constraint surface $\mathcal{C} \subset \mathcal{P}$,

$$
\left.F_{1}\right|_{\mathcal{C}}=\left.F_{2}\right|_{\mathcal{C}} .
$$

The surface $\mathcal{C}$ is determined by the constraints (47)-(48) and (51). An equivalence class of such functions, denoted by $F$, will be called a spacetime observable if it is invariant under Diff $\mathcal{M}$; i.e., if $F$ commutes on $\mathcal{C}$ with the generator $\mathcal{D}_{V}$,

$$
\left.\left\{F, \mathcal{D}_{V}\right\}\right|_{\mathcal{C}}=0,
$$

where $\mathcal{D}_{V}$ is given by (127). From the elementary functions $F^{g}:=g, F^{p}:=p, F^{Y}:=Y$ and $F^{P}:=P$ on $\mathcal{P}$, only the first three are non-trivial, because $F^{P}$ is equivalent to the zero function. From these, $F^{g}$ and $F^{p}$ are spacetime observables,

$$
\begin{aligned}
& \left.\left\{F^{g}, \mathcal{D}_{V}\right\}\right|_{\mathcal{C}}=0, \\
& \left.\left\{F^{p}, \mathcal{D}_{V}\right\}\right|_{\mathcal{C}}=0,
\end{aligned}
$$

while $F^{Y}=Y$ is not a spacetime observable,

$$
\left.\left\{F^{Y}, \mathcal{D}_{V}\right\}\right|_{\mathcal{C}}=V(Y) .
$$

In general, any dynamical variable on $\mathcal{P}$ that is independent of $Y$ is a spacetime observable. The Dirac observables form a subset of spacetime observables that are additionally invariant under the action of the generator $\mathcal{D}_{(\delta \mathbf{T}, \delta \mathbf{X})}$ of deformations. An equivalent description of the Dirac observables, which follows the standard definition, is that $F^{\text {Dir }}$ is a Dirac observable if it commutes on $\mathcal{C}$ with all first-class constraints:

$$
\begin{aligned}
\left.\left\{F^{\text {Dir }}, P_{\alpha}\right\}\right|_{\mathcal{C}} & =0, \\
\left.\left\{F^{\text {Dir }}, H\right\}\right|_{\mathcal{C}} & =0, \\
\left.\left\{F^{\text {Dir }}, H_{i}\right\}\right|_{\mathcal{C}} & =0 .
\end{aligned}
$$

Any such function also commutes with the Hamiltonian of (44). 


\subsection{The evolution of spacetime observables}

Each spacetime observable $F(g, p, Y, P)$ represents the Diff $\mathcal{M}$-invariant result of measuring an instantaneous canonical state of the system. Given any solution curve $\left(g_{(t)}, p_{(t)}, Y_{(t)}, P_{(t)}\right)$ in $\mathbb{R} \times \mathcal{C}$, the dynamical evolution of a spacetime observable $F$ can be defined by comparing the values of $F$ at various times $t$ along this particular solution

$$
F_{(t)}(g, p, Y, P):=F\left(g_{(t)}, p_{(t)}, Y_{(t)}, P_{(t)}\right)
$$

In other words, one compares the results of the same measurement at different times. Particular attention should be paid to the fact that $t$ is a Diff $\mathcal{M}$-invariant structure, according to equation (67). The evolution of the elementary spacetime observables $F^{g}:=g$ and $F^{p}:=p$ is given by

$$
\begin{aligned}
& F_{(t)}^{g}(g, p, Y, P)=F^{g}\left(g_{(t)}, p_{(t)}, Y_{(t)}, P_{(t)}\right)=g_{(t)}, \\
& F_{(t)}^{p}(g, p, Y, P)=F^{p}\left(g_{(t)}, p_{(t)}, Y_{(t)}, P_{(t)}\right)=p_{(t)} .
\end{aligned}
$$

Each Dirac observable $F^{\text {Dir }}$ is a spacetime observable that does not evolve in time,

$$
F_{(t)}^{\operatorname{Dir}}(g, p, Y, P)=F^{\operatorname{Dir}}\left(g_{(t)}, p_{(t)}, Y_{(t)}, P_{(t)}\right)=F^{\text {Dir }}(g, p, Y, P)
$$

on account of equations (145)-(147).

\subsection{The relation between observables and the sets $\Gamma$ and $\Delta$}

The connection between the spacetime observables, their subset of Dirac observables, and the sets $\Gamma$ and $\Delta$ of the extended action is as follows. Each element of the set of solutions of (44) defines a curve $\left(g_{(t)}, p_{(t)}, Y_{(t)}, P_{(t)}\right)$ in $\mathbb{R} \times \mathcal{C}$. As discussed in section 5 , any two solutions in this set that can be mapped onto each other by a spacetime diffeomorphism must have identical components $g_{(t)}$ and $p_{(t)}$ and differ only in their component $Y_{(t)}$. Hence, each element $\gamma$ of the set $\Gamma$ of Diff $\mathcal{M}$-classes of solutions of (44) is characterised by the solution-components $g_{(t)}$ and $p_{(t)}$. Therefore, in order to be able to distinguish between the elements of $\Gamma$ based on instantaneous measurements of the system, one has to measure the instantaneous data $g$ and $p$ at all times $t$. The resulting collection $\left(g_{(t)}, p_{(t)}\right), t \in \mathbb{R}$ of values then determines a unique element of $\Gamma$. Accordingly, the collection $\left\{\left(F_{(t)}^{g}, F_{(t)}^{p}\right)\right\}, t \in \mathbb{R}$, of the elementary spacetime observables is able to distinguish between the elements of $\Gamma$. Such a collection can be regarded as inducing a complete set of functions on $\Gamma$.

Referring to section 5 again, the set $\Delta$ of first-class orbits of (44) is derived from the set $\Gamma$ by identifying two elements $\gamma_{1}=\left(g_{1}, p_{1}\right)$ and $\gamma_{2}=\left(g_{2}, p_{2}\right)$ of $\Gamma$ that reconstruct via arbitrary choices of the foliation mapping $Y$ the same Diff $\mathcal{M}$-class $\{\mathbf{G}\}$ of spacetime solutions. In the spacetime representation, $\gamma_{1}$ and $\gamma_{2}$ are denoted by Diff $\mathcal{M}$-classes of solutions; i.e., $\gamma_{1}=$ $\left\{\left(\mathbf{G}_{1}, \mathbf{T}_{1}, \mathbf{X}_{1}\right)\right\}$ and $\gamma_{2}=\left\{\left(\mathbf{G}_{2}, \mathbf{T}_{2}, \mathbf{X}_{2}\right)\right\}$. Two such elements of $\Gamma$ define the same element $\delta \in \Delta$ if the metric classes $\left\{\mathbf{G}_{1}\right\}$ and $\left\{\mathbf{G}_{2}\right\}$ coincide. The mappings $\mathbf{T}$ and $\mathbf{X}$ are not involved at all 
in this last consideration. As a result, two elements $s_{1}=\left(\mathbf{G}_{1}, \mathbf{T}_{1}, \mathbf{X}_{1}\right)$ and $s_{2}=\left(\mathbf{G}_{2}, \mathbf{T}_{2}, \mathbf{X}_{2}\right)$ of the set of solutions of (44) define the same element $\delta \in \Delta$ if they can be mapped onto each other by a combined action of spacetime diffeomorphisms and deformations of the mappings $\mathbf{T}$ and $\mathbf{X}$. In phase space, this corresponds to a combined action of the generators $\mathcal{D}_{\mathcal{M}}$ and $\mathcal{D}_{(\delta \mathbf{T}, \delta \mathbf{X})}$. Any function on $\mathcal{P}$ that is invariant under the action of both these generators, commutes on $\mathcal{C}$ with all eight first-class constraints, and hence projects down to a function on $\Delta$. These considerations reexpress the standard result that Dirac observables induce functions on $\Delta$.

\subsection{On the problem of time}

As emphasised in the introduction, the covariant canonical formalism admits two interpretations. The conventional interpretation, of Bergmann and Dirac, treats all first-class constraints as gauge generators. This implies that the diffeomorphisms of $\mathcal{M}$ and the deformations of the mappings $\mathbf{T}$ and $\mathbf{X}$ have no measurable consequences. The fields $\mathbf{T}$ and $\mathbf{X}$ are regarded as physically unimportant, and only the subset $\Delta \subset \Gamma$ retains its physical significance. In this case, the fact that $t$ is a Diff $\mathcal{M}$-invariant parameter, in accordance with equation (67), is not sufficient to guarantee that it is a gauge-invariant parameter. This is because, in accordance with equation (96), $t$ is not invariant under a deformation of the mapping $\mathbf{T}$, which is also treated as a gauge transformation. Thus, the term observable is reserved for the Dirac observables, and the classical problem of time re-appears in its standard form. If this interpretation is accepted, the only surviving attribute of the extended action is its ability to accommodate the representations of the Lie algebra of Diff $\mathcal{M}$.

Actually, it is not only the Lie algebra, but the group itself, that can be represented within the phase space of (44). In particular, a conceptual difficulty which arose in the formalism of Isham and Kuchař does not arise here. This difficulty, analysed in Ref. [12], is that the space of spacelike embeddings is not an invariant submanifold of the space of all embeddings. Hence, the vector fields on the space of spacelike embeddings are not complete, and only the Lie algebra of spacetime diffeomorphisms can be represented in the phase space of [12]. This is not a problem here on account of the fact that the compatibility conditions are not imposed directly on the foliation variable $Y$. Instead, they are incorporated into the definition of the virtual fields $\left(g, N, N^{i}\right)$. Since the metric $\mathbf{G}$ and the mappings $\mathbf{T}$ and $\mathbf{X}$ all transform covariantly under spacetime diffeomorphisms, the fields $g, N$ and $N^{i}$ remain invariant under these diffeomorphisms, and the compatibility conditions incorporated in them are preserved. The fact that the vector fields $\delta Y$ do not need to be restricted is especially evident at the level of the variation of the action (44), which leaves the mapping $Y$ entirely undetermined.

This property of the covariant canonical formalism also solves the problem of interpreting the so-called microcausality condition in canonical quantum gravity. The microcausality condition, discussed, for example, by Isham in Ref. [41], is the requirement that quantum operators should commute for all spacetime points that are spacelike separated. As originally realised by Fredenhagen and Haag [67], for most pairs of points in spacetime there exists at least one 
Lorentzian metric with respect to which these points are not spacelike separated. Insofar as all metrics are virtually present in quantum theory, the microcausality consition is violated. However, as argued in Refs. [3]- [4, this problem is overcome provided that both the foliation and the spacetime metric transform covariantly, which is also the case here.

These points acquire physical significance once the alternative, and richer, interpretation of the formalism is accepted. Then, the entire set $\Gamma$ is considered physically meaningful, and the theory retains its full effect. The observable aspects of vacuum gravity are not limited to the Dirac observables, and spacetime observables arise whose dynamical evolution is non-trivial. In particular, for each physical state $\delta \in \Delta$, there corresponds a whole set $\{\gamma\}$ of states $\gamma \in \Gamma$, all of which are foliation-dependent but Diff $\mathcal{M}$-invariant and, hence, observable. Kuchař's terminology is then quite appropriate: Dirac observables were referred to as perennials in Ref. [39], precisely in order to separate them from other observable aspects of general relativity that evolve in time. Kuchař criticised the identification of the Hamiltonian with a gauge generator, and proposed certain evolving observables that do not weakly commute with it.

The spacetime observables derived from (44) represent such observable aspects of the vacuum theory. In particular, the elementary functions $F^{g}=g$ and $F^{p}=p$ on the phase space $\mathcal{P}$ are the prototypes of evolving, foliation-dependent, spacetime observables. Kuchařs proposal for observables in Ref. [39] does not entirely coincide with the present one, but corresponds to an intermediate viewpoint that regards the deformations of $\mathbf{X}$ as gauge, but those of $\mathbf{T}$ as measurable. According to this standpoint, the elementary functions $F^{g}=g$ and $F^{p}=p$ are not observables, but any Diff $\Sigma$-invariant functional of $g$ and $p$ is. Of course, accepting here $\mathbf{X}$ as a gauge field would imply an asymmetrical treatment of the mappings $\mathbf{T}$ and $\mathbf{X}$, and this would not be supported by the assignment of a reference system to each pair $(\mathbf{T}, \mathbf{X})$.

Finally, a comment can be made about the Diff $\mathcal{M}$-invariant time parameter $t$, upon which the interpretation of the covariant canonical formalism rests. Although the energy-momentum of the fields $\mathbf{T}$ and $\mathbf{X}$ vanishes in (30), and hence the time parameter $t$ of (44) cannot be regarded as being part of the system in the strict sense, this may not be such an undesired property after all. For this notion of time is not very distant from the notion of time arising in conventional quantum field theory - or in histories theory [2]- [4] as the parameter of partial ordering. There, too, time is external to the system, in the sense that it forms part of the background on which the system evolves, in accordance with the Copenhagen interpretation. Actually, the parameter $t$ of (44) is as external as a time can ever be in a closed system such as general relativity. This may be regarded as an indication that the covariant canonical action provides a link between the special and the general theory of relativity or, more generally, between a theory on a given background and gravitation theory. However, approaching quantum gravity in this light presupposes a quantisation method which, at the very least, should respect the distinction between the sets $\Gamma$ and $\Delta$. If such a method is attainable remains to be studied. 


\section{Acknowledgments}

I would like to thank Charis Anastopoulos, Petr Hájíček, Chris Isham, Karel Kuchař and Ntina Savvidou for reading the final draft of this paper.

\section{References}

[1] I. Kouletsis and K. V. Kuchař, Phys. Rev. D65, 125026 (2002).

[2] K. Savvidou, Class. Quant. Grav. 18, 3611 (2001).

[3] K. Savvidou, Class. Quant. Grav. 21, 615 (2004).

[4] K. Savvidou, Class. Quant. Grav. 18, 631 (2004).

[5] P. Dirac, Proc. Royal. Soc. of London A246, 326-332 (1958), and Proc. Royal. Soc. of London A246, 333-343 (1958).

[6] R. Arnowitt, S. Deser, and C. W. Misner, in Gravitation: An Introduction to Current Research, edited by L. Witten (Wiley, New York,1962) and relevant references therein.

[7] P. Bergmann, Rev. Mod. Phys. 33, 510 (1961).

[8] P. A. M. Dirac, in Lectures on Quantum Mechanics, Yeshiva Univ. Press, New York (1964).

[9] R. Abraham and J. E. Marsden, in Foundations of mechanics, 2nd ed., Benjamin/Cummings Publishing Co. Reading, Mass., (1978).

[10] Hájíček, Lect. Notes. Phys. 631, 255 (2003).

[11] C. Torre, Phys. Rev. D46, R3231 (1992).

[12] C. J. Isham and K. V. Kuchař, Ann. Phys. (N.Y) 164, 288; 316 (1985).

[13] K. V. Kuchař, Found. Phys. 16, 193-208 (1986).

[14] K. V. Kuchař and C. G. Torre, Phys. Rev. D43, 419 (1991).

[15] K. V. Kuchař and C. G. Torre, Phys. Rev D44, 3116 (1991).

[16] C. L. Stone and K. V. Kuchař, Class. Quantum Grav. 9, 757 (1991).

[17] K. V. Kuchař, Phys. Rev. D44, 43 (1992).

[18] J. D. Brown and K. V. Kuchař, Phys. Rev. D51, 5600 (1995).

[19] K. V. Kuchař and J. D. Romano, Phys. Rev. D51, 5579 (1995). 
[20] I. Kouletsis, Class. Quant. Grav. 13, 3085 (1996).

[21] J. Bičák and K. V. Kuchař, Phys. Rev. D56, 4878 (1997).

[22] R. B. Griffiths, J. Stat. Phys. 36, 219 (1984).

[23] R. Omnès, J. Stat. Phys. 53, 893; 933; 957 (1988).

[24] R. Omnès, J. Stat. Phys. 57, 357 (1989).

[25] R. Omnès, Ann. Phys. (N.Y.) 201, 354 (1990).

[26] R. Omnès, Rev. Mod. Phys. 64, 339 (1992).

[27] M. Gell-Mann and J. Hartle, in Proceedings of the Third International Symposium on the Foundations of Quantum Mechanics in the Light of New Technology, (Physical Society of Japan, Tokyo, Japan), 321 (1990)

[28] M. Gell-Mann and J. Hartle, in Complexity, Entropy and the Physics of Information, SFI Studies in the Science of Complexity, Vol. VIII, (Addison-Wesley, Reading), 425 (1990)

[29] M. Gell-Mann and J. Hartle, in Proceedings of the 25th International Conference on High Energy Physics, (World Scientific, Singapore) (1990)

[30] J. Hartle, in Quantum Cosmology and Baby Universes, eds S. Coleman, J. Hartle, T. Piran and S. Weinberg (World Scientific, Singapore) (1991)

[31] J. Hartle, in Gravitation and Quantizations: Les Houches 1992-Session LVII, eds B. Julia and J. Zinn-Justin (Elsevier Science, Netherlands), 1 (1995)

[32] C. J. Isham, J. Math. Phys. 35, 2157 (1994).

[33] C. J. Isham and N. Linden, J. Math. Phys. 35, 5452 (1994).

[34] C. J. Isham and N. Linden, J. Math. Phys. 36, 5392 (1995).

[35] C. J. Isham, N. Linden, K. Savvidou and S. Schreckenberg, Jour. Math. Phys. 39, 1818 (1997).

[36] K. Savvidou, J. Math. Phys. 40, 5657 (1999).

[37] K. Savvidou, Continuous time in consistent histories, PhD thesis, University of London, gr-qc/9912076 (1999).

[38] I. Kouletsis, Classical Histories In Hamiltonian Systems, PhD thesis, University of London, gr-qc/0108021 (2001). 
[39] K. V. Kuchař, in General Relativity and Gravitation, eds. Gleiser, Kozameh, Moreschi (1993).

[40] C. J. Isham and K. Savvidou, J. Math. Phys 43 (2001).

[41] C. J. Isham, Lectures presented at NATO Advanced Study Institute, Salamanca gr-qc/9210011 (1992)

[42] K. V. Kuchar, in Proceedings of the 4th Canadian Conference on General Relativity and Relativistic Astrophysics, World Scientific, Singapore (1992)

[43] S. Carlip, Rept. Prog. Phys. 64, 885 (2001)

[44] T. Thiemann, gr-qc/0110034 (2001)

[45] J. J. Halliwell, gr-qc/0208018 (2002)

[46] D. C. Salisbury, Mod. Phys. Lett A18 2475 (2003)

[47] T. P. Shestakova and C. Simeone, Grav. Cosmol. 10, 161 (2004)

[48] J. M. Pons and D. C. Salisbury, Phys. Rev. D71 124012 (2005)

[49] D. Hilbert, 2 Mitt., Nachr. Ges. Wiss. Göttingen 53 (1917). Included in David Hilbert gessammelte Abhandlungen, Vol. 3, 2nd ed. (Springer, Berlin, 1970).

[50] A. Einstein, Ueber die spezielle und die allgemeine Relativitätstheorie (Vieweg, Braunschweig, 1920), Ch. 28, p. 67; English transl. R. W. Lawson, Relativity: The Special and the General Theory, 17th ed. (Crown, New York, 1961).

[51] J. Stachel, in Proc. 11th Int. Conf. on General Relativity and Gravitation, Cambridge University Press, Stockholm (1986)

[52] C. Rovelli, in Class. Quantum Grav. 8, 297-316 (1991).

[53] R. Geroch, in Journ. Math. Phys. 11, 437-509 (1970)

[54] P. Hájíček and Kijowski, gr-qc/9908051 (1999)

[55] A. E. Fischer and J. E. Marsden, in General Relativity. An Einstein Centenary Review, eds. S. W. Hawking and W. Israel, Cambridge University Press, Cambridge, 1979.

[56] M. Henneaux and C. Teitelboim, in Quantization of Gauge Systems Princeton University Press, Princeton (1992)

[57] J. M. Pons, Stud. Hist. Philos. Mod. Phys 36491 (2005) 
[58] E. Kretschmann, in Ann. Phys. (Lpz.) 53, 575-614 (1917).

[59] E. Cartan, in La Théorie des groupes et la géometrie, L' Enseignement Math. 26, 200 (1927)

[60] V. Fock, in The theory of Space, time and gravitation, Pergamon Press, Oxford (1956)

[61] J. L. Anderson, in Recent Developments in General Relativity, Pergamon Press (1962)

[62] K. V. Kuchař, in Highlights in Gravitation and Cosmology, Iyer, Kembhavi, Narlikar, Vishveshwara eds. (1988)

[63] R. Sorkin, Mod. Phys. Lett. A 17, No 11, 695 (2002)

[64] I. M. Anderson and C. G. Torre, Commun. Math. Phys 176479 (1996)

[65] J. B. Pitts and W. C. Schieve, Found. Phys. 34, 211 (2004)

[66] P. G. Bergmann and A. Komar, Int. J. Phys. 5, 15 (1972)

[67] K. Fredenhagen and R. Haag, in Comm. Math. Phys. 108, 91-115 (1987). 